\title{
Lactobacillus rhamnosus GG modifies the metabolome of pathobionts in gnotobiotic mice
}

Jinhee Kim ${ }^{1 \dagger}$, Iyshwarya Balasubramanian ${ }^{2 \dagger}$, Sheila Bandyopadhyay ${ }^{2}$, lan Nadler ${ }^{1}$, Rajbir Singh ${ }^{2}$, Danielle Harlan ${ }^{1}$, Amanda Bumber ${ }^{3}$, Yuling He $\mathrm{H}^{4,5}$, Lee J. Kerkhof ${ }^{6}$, Nan Gao ${ }^{2}$, Xiaoyang Su ${ }^{4}$ and Ronaldo P. Ferraris ${ }^{1 *}$

\begin{abstract}
Background: Lactobacillus rhamnosus GG (LGG) is the most widely used probiotic, but the mechanisms underlying its beneficial effects remain unresolved. Previous studies typically inoculated LGG in hosts with established gut microbiota, limiting the understanding of specific impacts of LGG on host due to numerous interactions among LGG, commensal microbes, and the host. There has been a scarcity of studies that used gnotobiotic animals to elucidate LGG-host interaction, in particular for gaining specific insights about how it modifies the metabolome. To evaluate whether LGG affects the metabolite output of pathobionts, we inoculated with LGG gnotobiotic mice containing Propionibacterium acnes, Turicibacter sanguinis, and Staphylococcus aureus (PTS).
\end{abstract}

Results: $16 \mathrm{~S}$ rRNA sequencing of fecal samples by lon Torrent and MinION platforms showed colonization of germfree mice by PTS or by PTS plus LGG (LTS). Although the body weights and feeding rates of mice remained similar between PTS and LTS groups, co-associating LGG with PTS led to a pronounced reduction in abundance of $P$. acnes in the gut. Addition of LGG or its secretome inhibited $P$. acnes growth in culture. After optimizing procedures for fecal metabolite extraction and metabolomic liquid chromatography-mass spectrometry analysis, unsupervised and supervised multivariate analyses revealed a distinct separation among fecal metabolites of PTS, LTS, and germ-free groups. Variables-important-in-projection scores showed that LGG colonization robustly diminished guanine, ornitihine, and sorbitol while significantly elevating acetylated amino acids, ribitol, indolelactic acid, and histamine. In addition, carnitine, betaine, and glutamate increased while thymidine, quinic acid and biotin were reduced in both PTS and LTS groups. Furthermore, LGG association reduced intestinal mucosal expression levels of inflammatory cytokines, such as IL-1a, IL-1 $\beta$ and TNF-a.

Conclusions: LGG co-association had a negative impact on colonization of $P$. acnes, and markedly altered the metabolic output and inflammatory response elicited by pathobionts.

Keywords: Competitive exclusion, Fecal metabolites, Germ-free mice, Inflammation, Liquid chromatography, mass spectrometry, Microbiota, Propionibacterium acnes

\footnotetext{
* Correspondence: ferraris@njms.rutgers.edu

${ }^{\dagger}$ Jinhee Kim and lyshwarya Balasubramanian contributed equally to this work.

${ }^{1}$ Department of Pharmacology, Physiology and Neurosciences, Medical

Science Building, New Jersey Medical School, Rutgers University, Newark, NJ 07103, USA

Full list of author information is available at the end of the article
}

C C The Author(s). 2021 Open Access This article is licensed under a Creative Commons Attribution 4.0 International License, which permits use, sharing, adaptation, distribution and reproduction in any medium or format, as long as you give appropriate credit to the original author(s) and the source, provide a link to the Creative Commons licence, and indicate if changes were made. The images or other third party material in this article are included in the article's Creative Commons licence, unless indicated otherwise in a credit line to the material. If material is not included in the article's Creative Commons licence and your intended use is not permitted by statutory regulation or exceeds the permitted use, you will need to obtain permission directly from the copyright holder. To view a copy of this licence, visit http://creativecommons.org/licenses/by/4.0/. The Creative Commons Public Domain Dedication waiver (http://creativecommons.org/publicdomain/zero/1.0/) applies to the data made available in this article, unless otherwise stated in a credit line to the data. 


\section{Background}

Lactobacillus rhamnosus GG (LGG) is a Gram-positive facultative anaerobic bacterium of the phylum Firmicutes typically associated, as are many other Lactobacillus spp., with the treatment and prevention of intestinal inflammatory disorders [1-3]. Amounts of endogenous Lactobacillus spp. are significantly reduced in the intestinal lumen of colitis patients [4]. The LGG strain (ATCC 53103) is the most widely used probiotic species sold under different trademarks [5]. LGG is thought to upregulate the synthesis of anti-inflammatory cytokines and downregulate the production of pro-inflammatory cytokines in a variety of gut-associated disorders [6, 7], but the mechanisms are unknown. The antiinflammatory characteristics of LGG could be due to the bacterial structure itself as well as to its secreted proteins or metabolites. Heat-killed Lactobacillus bulgaricus and cell-surface modified LGG attenuate dextran sulfate sodium (DSS)-induced colitis in mice [8, 9], suggesting that cell surface characteristics underlie at least some of their probiotic properties. Mucus-binding appendages like SpaCBA pili and mucus-binding surface adhesins such as MabA and MBF play a role not only in promoting intestinal colonization by LGG but also in LGG's anti-inflammatory and immunomodulatory effects $[10,11]$. The two secreted LGG proteins, p40 and p75, inhibit apoptosis in mouse intestinal epithelial cells treated with the pro-inflammatory cytokine tumor necrosis factor-alpha (TNF- $\alpha$ ), mainly by reinforcing the tight junction barrier, thereby enhancing epithelial integrity $[12,13]$. Surprisingly, there has been a scarcity of studies investigating LGG-associated gut microbial metabolites. This is unfortunate, as Lactobacillus spp. may produce metabolites that can act as anti-inflammatory mediators. For example, Aryl Hydrocarbon Receptor (AHR) ligands from dietary and Lactobacilli-metabolized tryptophan utilized AHR signaling to mediate their beneficial effects, including exclusion of pathobiont species and mucosal protection from inflammation $[14,15]$.

A large number of studies of LGG and other Lactobacillus spp. incorporated bacterium in feeds or drinking water [1-3, 16-19]. In these studies, a Lactobacillus species was typically inoculated in a host that housed an established commensal microbiota [20]. Results from these studies reflected an outcome from interactions among Lactobacillus, numerous existing microbes, and the host, with limited insight on the specific impact of Lactobacillus. In contrast, more recent studies used Lactobacillus mono-association in gnotobiotic animals to investigate Lactobacillus-host interactions [21-24]. Although these mono-association approaches appeared to be more specific in elucidating Lactobacillus-host interactions, the mucosal environment completely lacked the presence of other microbial components, thereby limiting the discovery of physiological insights. In our experiment, we analyzed the effect of a Lactobacillus probiotic on gnotobiotic mice housing common commensal bacteria typically ingested by humans along with food which, more importantly, also cause gastrointestinal illnesses under certain conditions $[25,26]$.

Propionibacterium acnes is a slow-growing facultative anaerobic Gram-positive bacterium found in the gut and skin, and is considered to be a pathobiont as it plays a pivotal role in promoting gastrointestinal disorder and acne, respectively, during dysbiosis [27-29]. While relatively benign, $P$. acnes was an important opportunistic pathogen causing implant-associated infections, and thought to initiate chronic lymphocytic gastritis [30]. Patients with a high abundance of Helicobacter pylori, P. acnes and $P$. copri were at a higher risk of developing gastric cancer [31]. Turicibacter sanguinis is a strictly anaerobic, Grampositive, rod-shaped commensal bacterium found in the gut and feces of many animals [32, 33]. T. sanguinis was associated with high levels of an antimicrobial fatty acid, butyrate, and of a proinflammatory cytokine TNF in the gut $[34,35]$ and was recently discovered to express a sodium symporter protein that absorbed the neurotransmitter serotonin (5-hydroxytryptamine) [36]. Interestingly, it is depleted in dogs with inflammatory bowel disease [33]. In hosts associated with $T$. sanguinis, the bacteria affected lipid and steroid metabolism, with corresponding reductions in host systemic triglyceride levels and adipocyte size [36]. Commonly responsible for numerous cases of food poisoning [37], Staphylococcus aureus is a facultative anaerobic Gram-positive, round-shaped, commensal bacterium that is frequently found in the upper respiratory tract and the skin. The human intestinal tract is commonly colonized by $S$. aureus and it is unclear how this bacteria becomes virulent as to cause serious outbreaks of gastrointestinal infections $[38,39]$. S. aureus does produce a heat-stable enterotoxin which, within hours after bacterial ingestion, causes severe intestinal inflammation resulting in abdominal cramping and diarrhea [40].

In this study, we tested the hypothesis that LGG coassociation modifies not only the metabolome but also the mucosal inflammatory response to a pathobiont community. We compared mice with $P$. acnes, T. sanguinis and S. aureus (PTS) to mice additionally inoculated with LGG (LTS). We demonstrated robust modulatory effects of LGG on colonization by pathobiont bacteria, their metabolome output, and host intestinal inflammation.

\section{Results}

Fecal metabolite extraction normalization minimized inter-sample variations

Fecal metabolomic analysis has been increasingly used to identify and characterize host-microbe interaction 
associated with metabolite profiles. However, a challenge has been the large inter-sample variabilities that may be attributed to procedural and other factors unrelated to host or microbial pathways of interest. These include different extraction methods, variable amounts of fecal material, and batch-to-batch sampling discrepancies. Previous studies have shown that extraction of mouse feces and subsequent normalization played a role in determining the quality of analysis [41, 42]. The efficacies of various extraction protocols have been compared in earlier studies [43, 44]. However, even when a unified extraction methodology [43] was followed, we noted considerable variations across samples. We thus designed a series of experiments to gauge the amount of variabilities attributable to sample sizes and collection batches, with the overarching goal of developing an optimal LC-MS method, which, upon minimizing nonbiological variabilities, allowed for a robust and reproducible identification of metabolic features characteristic of host-microbe interaction.

We used fecal samples from GF mice to minimize the biological variabilities prominently caused by the versatile microbial community. Age-matched GF C57/BL6 mice were housed in individual cages $(n=3)$ in a gnotobiotic facility and fed with an autoclavable diet throughout the study (Supplemental Table 1A). Over a period of 9 weeks, 7 batches of fecal samples were longitudinally collected from each mouse, weighted, and snap-frozen for storage. We chose to use LC-MS due to higher detection sensitivity and broader coverage of polar metabolites compared to other metabolomics tools such as nuclear magnetic resonance [45]. Therefore, global metabolomic profile was determined by targeted LC-MS in both positive and negative ion modes (Fig. 1a).

A batch of 21 fecal samples (7 samples per mouse) of different sizes were extracted and analyzed by LC-MS. Without normalization of the abundance of metabolites against fecal weight, PCA did not identify any noticeable clustering, even among samples collected from the same animal (Fig. 1b), illustrating the considerably large sampling variabilities. We then applied an universal approach of sampling normalization developed by $\mathrm{Xu}$ et al. [46] in which metabolite concentration was normalized to the weight of the respective fecal sample at different collection days after LC-MS analysis (referred to as post-LC-MS normalization). The normalized PCA plot showed that 18 out of 21 fecal extracts exhibited tight clustering among samples collected from the same animal at a different collection day (Fig. 1c). The 3 outliers (one from each mouse) were samples obtained on the same collection day and their average fecal weight was only $15-40 \%$ of the samples from the other 6 sampling days. This suggested that the fecal sample weight contributed to variations in metabolic profile. Otherwise, the fecal metabolic profile of samples from male and female mice post LC-MS normalization (compare mouse 3 with 1 and 2, Fig. 1c) was the same. We thus performed our subsequent experiments using only female GF mice.

To determine whether accounting for sample weight differences during metabolite extraction would reduce sample-to-sample variations, we longitudinally collected fecal samples from 10 female GF mice housed in 2 separate cages ( $n=5$ per cage). We normalized the extraction buffer volumes to each sample's weight during metabolite extraction (referred to as pre-LC-MS normalization) (Fig. 1d, e), resulting in extracts having concentrations around $13.33 \mathrm{mg}$ feces $/ \mathrm{ml}$. A total of 30 GF fecal samples were analyzed by LC-MS (3 longitudinal collections per mouse, $n=10$ ). Density plots of coefficient of variation (CV) were generated to visualize and compare the distribution in non-normalized, postLC-MS, and pre-LC-MS analyses (Fig. 1f). The pre-LCMS normalization showed the lowest mean CV of 0.493 compared to non-normalization (mean CV of 0.529) and post-LC-MS normalization (mean CV of 0.675 ), suggesting a greatly reduced variance across all samples. Heat map analysis of the distribution of metabolites nonnormalized (Fig. 1g), post-LC-MS normalized (Fig. 1h), and pre-LC-MS normalized (Fig. 1i) conditions illustrated a reduced variance across samples under pre-LCMS normalization. Thus, metabolite extraction normalized to fecal weight was proven to be an optimal approach in terms of suppressing variations in metabolomic analysis by LC-MS.

\section{LGG displaces pathobiont bacteria}

Based on the aforementioned optimization of extraction and analysis, we next determined the impact of LGG on the metabolomic output of $P$. acnes, T. sanguinis, and $S$. aureus. Fecal samples were longitudinally collected before and after bacterial association by gavage (Fig. 2a and Supplemental Fig. 1A) from 10 GF mice $(n=5$ per cage) fed a double-irradiated diet (Supplemental Table 1B). One cage of GF mice $(n=5)$ contained mostly PTS, while the other cage of GF mice was colonized with LGG in addition to the PTS (referred to as "LTS" group). 16S rRNA sequencing by Ion Torrent showed mice either had PTS and LTS groups (Fig. 2b-c and Supplemental Fig. 2A-D). The body weights and feeding rates of mice remained similar pre- and postgavage between PTS and LTS groups indicating that fecal metabolite quantities between groups were not confounded by these factors (Supplemental Figs. 1B-C).

Quantitative PCR with 16S rRNA-specific primers revealed similar levels of bacterial colonization in PTS and LTS mice (Fig. 2d). Using LGG-specific primers, successful colonization was confirmed in LTS mice (Fig. 2e). There was no LGG in PTS mice. P. acnes levels increased 


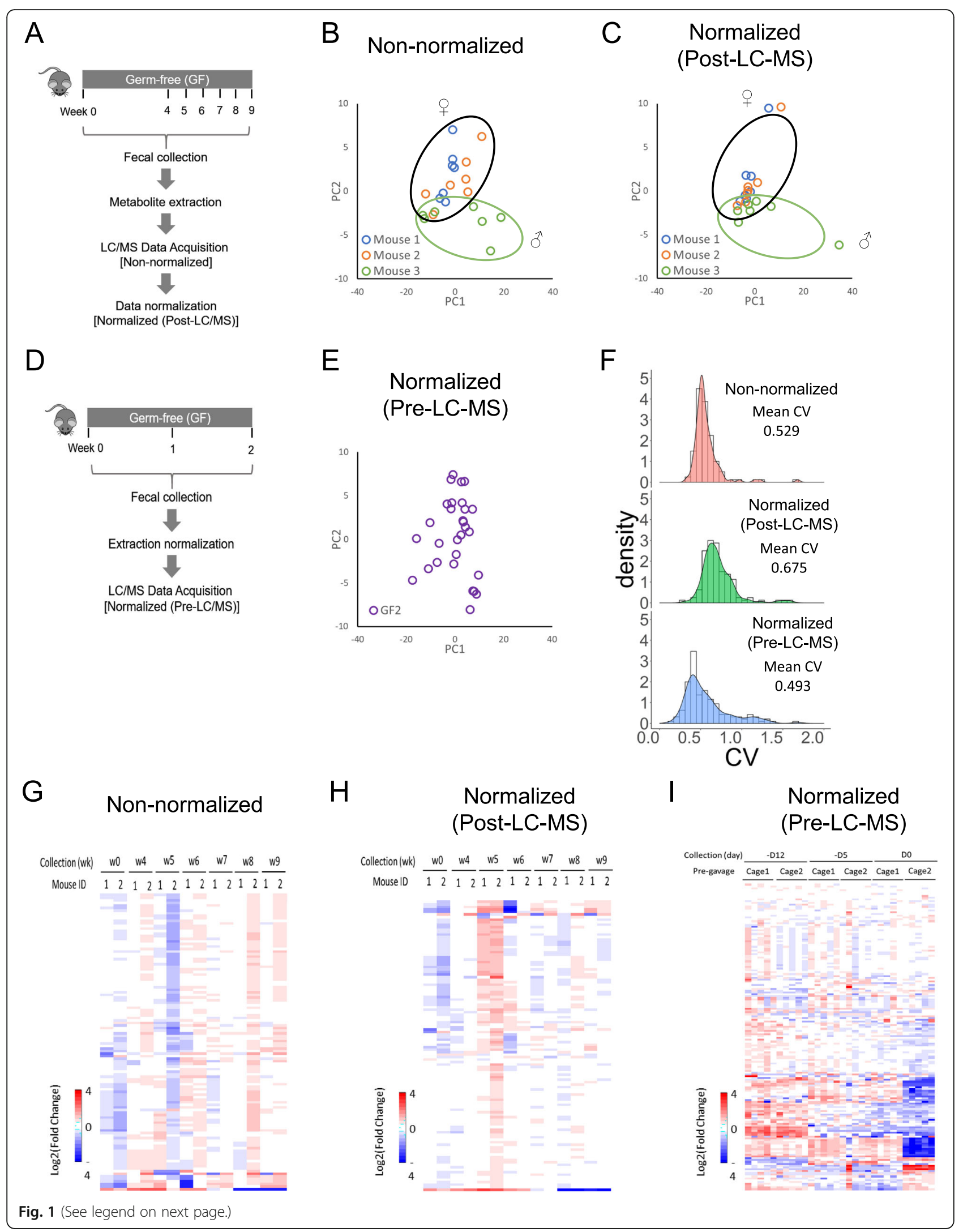




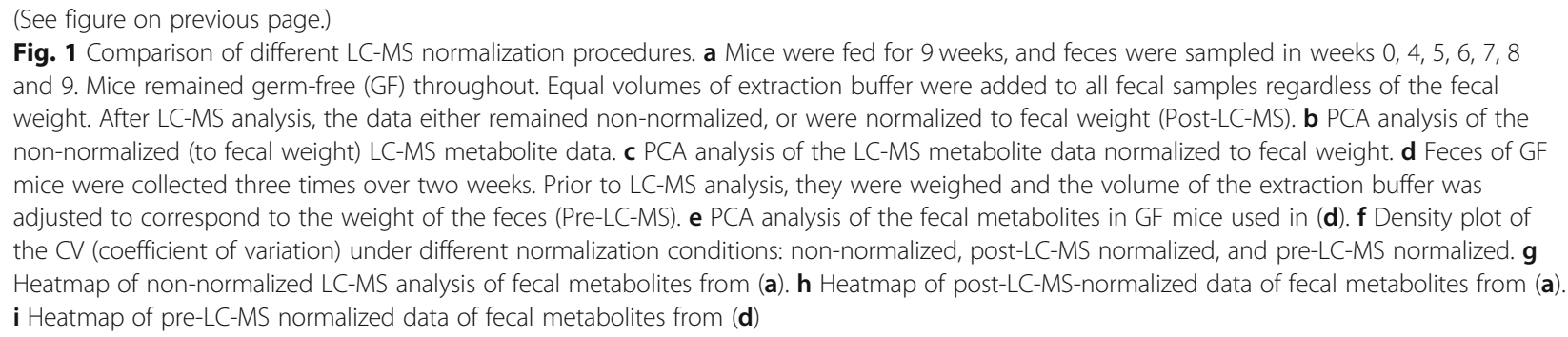

$>\sim 400,000$-fold over 2 weeks post-gavage in PTS mice but were only present in negligible numbers in LTS mice (Fig. 2f). Addition of LGG clearly led to a pronounced reduction in the relative abundances of Turicibacter and Propionibacterium (Fig. 2c and Supplemental Fig. 2A-D), indicating an LGG-mediated exclusion of these bacteria. We confirmed the inhibitory effect of LGG on P. acnes in vitro, as this species was virtually eliminated by LGG whereas T. sanguinis and S. aureus were not. LGG interacted with $P$. acnes leading to a $66.5 \%$ coaggregation, compared to $6.23 \%$ auto-aggregation by $P$. acnes (Fig. 2g-h).

\section{LGG modified fecal metabolomic output of pathobionts}

We then used LC-MS to examine fecal metabolites of PTS mice. Both unsupervised multivariate PCA (Supplemental Fig. 3) and supervised PLS-DA demonstrated a distinct separation between metabolites of PTS mice and pre-gavage GF mice (Fig. 3a), between those of LTS and GF mice (Fig. 3b), and between those of PTS and LTS mice (Fig. 3c). These data when analyzed further to determine significant variable's important in the projection (VIP) scores as discussed in the next section below, suggested a global alteration of metabolic output by PTS after LGG is administered in vivo.

By performing enrichment and pathway analysis using MetaboAnalyst 4.0 [47], we examined the metabolic pathways altered by PTS as well as those impacted by LGG coassociation. Compared to pre-gavage GF mice, PTS significantly upregulated ammonia recycling, glycine and serine metabolism, urea cycle, methylhistidine metabolism, arginine and proline metabolism, spermidine and spermine biosynthesis, as well as aspartate metabolism, while downregulating alanine metabolism, glucose-alanine cycle, urea cycle, purine metabolism, as well as glycine and serine metabolism. Compared to PTS, addition of LGG significantly upregulated 7 and downregulated 9 metabolic pathways (Fig. 3d, e, f). Several pathways are both upregulated and downregulated, suggesting that individual reactions within these pathways can be independently regulated to increase or decrease with LTS conditions.

\section{LGG alters metabolic prolife of PTS mice}

The alteration of metabolomic profile by LGG was strongly corroborated by the heat map visualization of polar metabolites (Fig. 3g). VIP analysis revealed 43 unique metabolites that had > 1.2 VIP scores in the GF vs. PTS comparison, 47 in the GF vs. LTS comparison, and 33 in PTS vs. LTS comparison (metabolites that appeared in both positive and negative ionization modes were counted only once). The selection of VIP cut-off score of $>1.2$ was based on discriminatory power when VIP $>1$ was considered influential [48]. By directly comparing PTS to LTS, we identified the top 10 metabolites with the highest VIP scores (Fig. 3h) that were either up-regulated or down-regulated in LTS (Supplemental Table 3A). We also identified the top 10 metabolites most significantly correlated with PTS (Fig. 3i, Supplemental Table 3B) or with LTS (Fig. 3j, Supplemental Table 3C) colonization of a GF condition. Metabolites that exhibit high (>1.2) VIP scores fall into four patterns: those that increase in both PTS and LTS, diminish in both PTS and LTS, decrease mainly in LTS, and increase primarily in LTS (see Supplemental Table 3A,B,C). An important summary of the comparisons of VIP scores between GF and PTS, between GF and LTS, as well as between LTS and PTS, is shown (Supplemental Table 3D).

\section{Metabolites upregulated with PTS and LTS}

Fecal metabolites found in high levels in both PTS and LTS groups may arise from bacterial synthesis of dietary precursors, bacterial modification of dietary metabolites, or from intestinal excretion by the host into the lumen. These include the amino acids betaine, carnitine and glutamate as well as the amino acid derivative dimethylL-arginine, all of which exhibit the highest VIP ranks and scores in all three comparisons of PTS and GF, of LTS and GF, as well as of PTS and LTS (Supplemental Tables 3A-D). These metabolites increase dramatically in fecal levels in both PTS and LTS compared with the pre-gavage GF condition (Fig. 4a). Other physiologically important metabolites found in high levels in the feces of PTS and LTS mice were the amino acids aspartate and histidine, vitamin $\mathrm{B}_{3}$ (nicotinate), and the polyamine spermine, which have VIP scores of 1.5-1.7 in both PTS and LTS (Supplemental Table 3A-D). Carnitine, betaine, glutamate, $\mathrm{N}$-acetyl-glutamate, histidine, nicotinate, dimethyl-L-arginine and spermine were also present in the diet, but clearly increased markedly in fecal levels 
A

Bacterial association

(Pathobiont mixture +/- LGG)

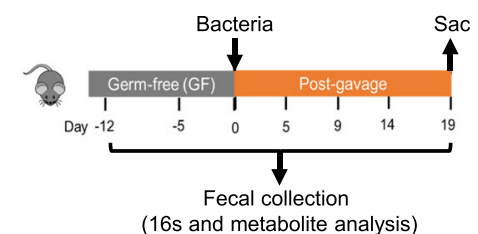

C

Genus

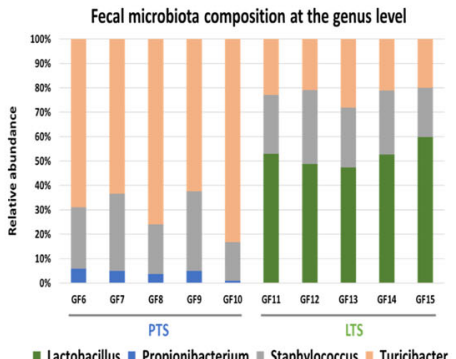

E

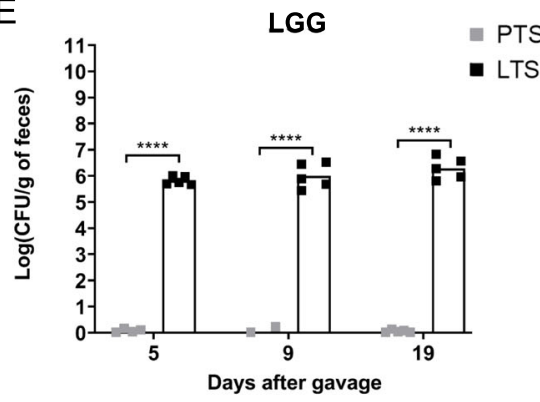

G

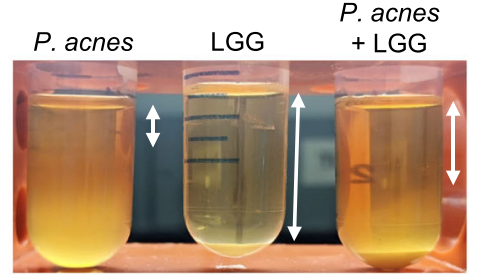

B Beta-diversity

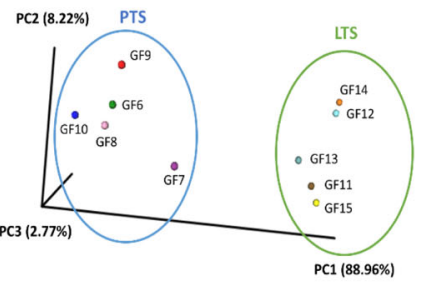

D

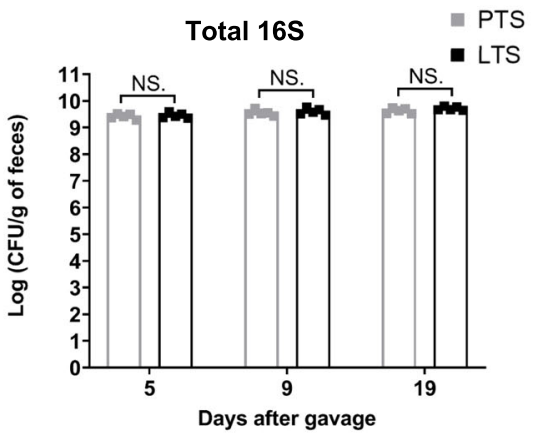

$\mathrm{F}$

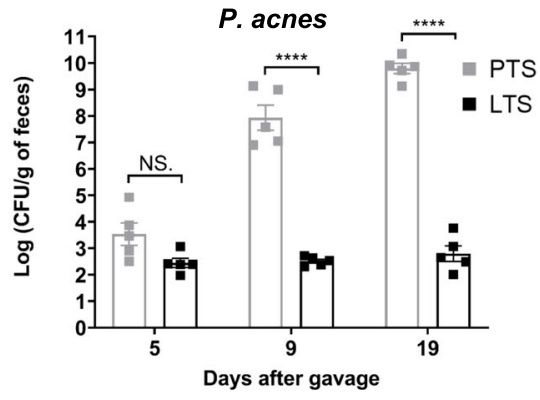

$\mathrm{H}$

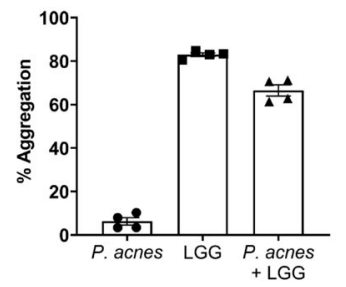

Fig. 2 LGG modifies bacterial and fecal metabolite composition of PTS. a Schematic diagram of the experimental design. Mice remained GF for 12 days, and on day 0 , bacteria (PTS or LTS) were gavaged then feces were sampled on days 0 (before gavage), 5, 9, 14 and 19. Metabolites from feces were extracted using the pre-LC-MS procedure outlined above, then subjected to 165 metagenomic sequencing and metabolomics. $\mathbf{b}$ Principal coordinate analysis (PCOA) indicating beta diversity of bacterial communities in PTS and LTS mice after gavage. Each point refers to fecal samples in specific mice, with GF6 to 10 mice in the PTS cage, and GF11 to 15 in the LTS cage. c Microbiota composition (relative OTU abundance) at the genus level of feces collected 14th day postgavage from PTS and LTS mice. d Abundance of 16S rRNA (d), LGG (e), and P. acnes (f) in the feces of PTS and LTS mice ( $n=5$ in each group) 5, 9, and 19 days after gavage. $\mathbf{g}$ Images of aggregation of $P$. acnes alone, LGG alone, and $P$. acnes and LGG in medium after $48 \mathrm{~h}$ of incubation. Percentage of autoaggregation of $P$. acnes and LGG and coaggregation of $P$. acnes with LGG (h) after $48 \mathrm{~h}$ of incubation

after colonization by both the bacterial groups (Fig. 4a). $\mathrm{N}$-acetyl-glutamate was not found in the diet and is likely a major product of bacterial or host metabolism. $\mathrm{N}$-acetyl-glutamate synthase, known to synthesize $\mathrm{N}$ acetylglutamate from glutamate and acetyl-CoA, is expressed by many bacteria and the mammalian small intestine [49].

\section{Metabolites downregulated with PTS and LTS colonization}

Fecal metabolites found in low concentrations in both PTS and LTS groups when compared to pre-gavage conditions might be reduced by bacterial catabolism (Fig. $4 \mathrm{~b})$. The nucleosides thymidine, inosine and guanosine, the nucleotide derivative 5-methylcytosine, the vitamin $\mathrm{B}_{7}$ biotin and the plant polyol quinic acid (VIP score 


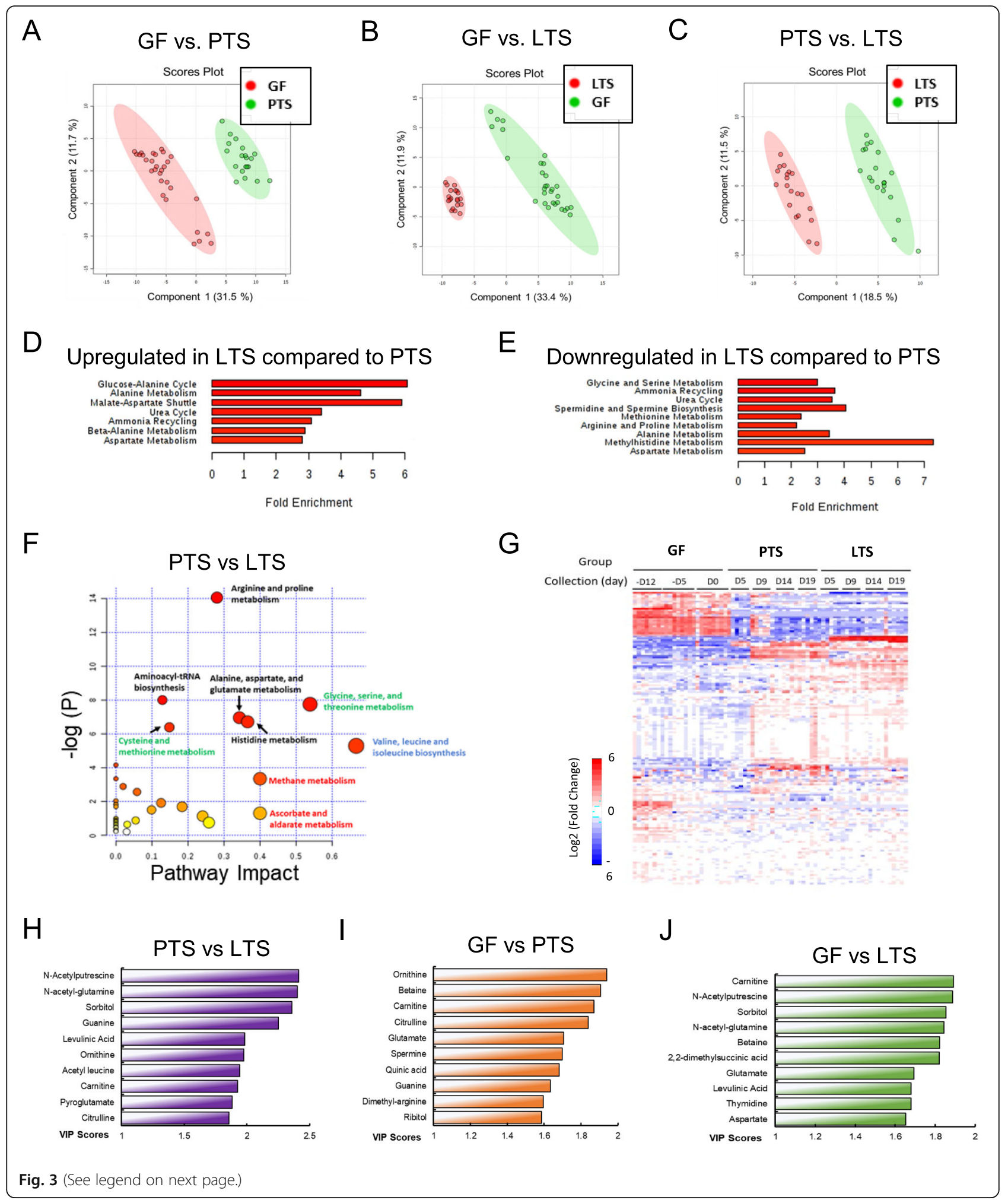

1.4 to 1.7) were highly downregulated in PTS and LTS groups compared to pre-gavage. Quinic acid is abundant in plants, but could also be produced from corn starch which constitutes $\sim 55 \%$ of our purified diet, by irradiation [50] (Supplemental Table 1B). Quinic acid is readily metabolized by the gut microbiota [51], explaining reduced concentrations in the feces of PTS and LTS groups relative to pre-gavage. 


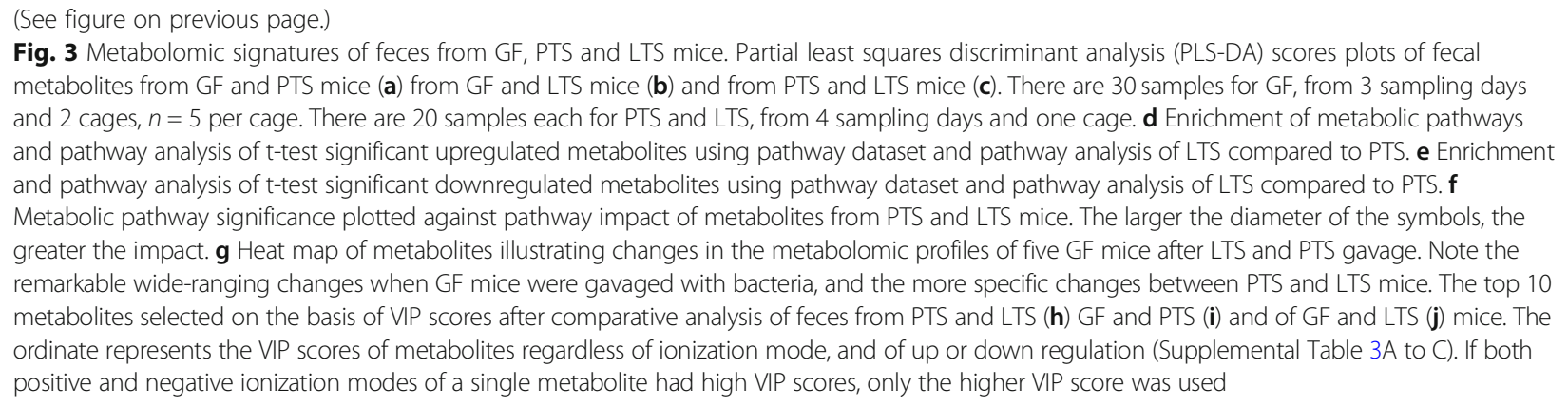

\section{LGG diminished PTS-derived metabolites}

Ornithine, citrulline, guanine (Fig. 4c) and isoleucine (not shown) were synthesized abundantly by PTS then were strongly catabolized by LGG. Sorbitol and pyruvate were catabolized from the diet by both PTS and LTS, but LGG greatly enhanced this catabolic activity (Fig. 4c; Supplemental Table 3D).

\section{Metabolites uniquely upregulated by LGG colonization}

Levels of $\mathrm{N}$-acetyl-putrescine, $\mathrm{N}$-acetyl-glutamine (Fig. 4d) and indolelactic acid (not shown) were similar between PTS and pre-gavage, suggesting none of the PTS bacteria were able to synthesize these metabolites (Supplemental Tables 3A-D). Their levels increased markedly in LTS mice, resulting in high VIP scores of $>1.7$. This suggested their collective importance to the distinctive metabolome of LTS. Levulinic acid, a by-product of the gut neurotransmitters histamine and cellulose, (Fig. 4d) as well as the fatty acid 2,2-dimethylsuccinic acid (not shown) also had high VIP scores in LTS group (Supplemental Table 3A-D). These fecal metabolites increased modestly in PTS, but were highly upregulated in LTS mice, suggesting that LGG colonization markedly enhanced their production compared to PTS. Other fecal metabolites found in high levels in LTS compared to PTS and pre-gavage were amino acid derivatives acetyl leucine and pyroglutamate, the pentose alcohol ribitol, and purine derivative hypoxanthine.

\section{Unidentified metabolite features in PTS and LTS mice}

In addition to the targeted metabolomics data analysis, we performed untargeted analysis to reveal more metabolomics features that were impacted by bacterial colonization. Similar to the results from the targeted analysis, we found features that fall into four categories as described earlier (Fig. 5a-d). The exact identities of these metabolites were not known. However, based on the accurate mass and $\mathrm{MS}^{2}$ spectra (Supplemental Fig. 4, Supplemental Table 4), we suspect one metabolite ([M$\mathrm{H}]^{-} \mathrm{m} / \mathrm{z}$ 163.0607, retention time $3.68 \mathrm{~min}$ ) that was upregulated in both LTS and PTS groups to be deoxyhexose. Another metabolite $\left([\mathrm{M}-\mathrm{H}]^{-} \mathrm{m} / \mathrm{z}\right.$ 357.1028, retention time $9.68 \mathrm{~min}$ ) that is downregulated in LTS group could be a sugar acid $\mathrm{C}_{12} \mathrm{H}_{22} \mathrm{O}_{12}$ (Fig. 5e-f, Supplemental Table 4). These results suggested that carbohydrate metabolism was significantly impacted by the LTS and PTS colonization.

\section{LGG suppressed some inflammatory features of the pathobionts}

As PTS bacteria were implicated in gastrointestinal pathogenesis, we next examined the impact of LGG on PTS-elicited mucosal immune responses by evaluating various inflammation-related genes in proximal and distal small intestines and colons. Interestingly, the expression levels of IL-22 and IL-22R were both significantly down-regulated in the mucosa of all intestinal regions in LTS group (Fig. 6a and b, Supplemental Fig. 5), suggesting LGG-mediated suppression of IL-22 signaling.

The pro-inflammatory cytokine, TNF- $\alpha$, was significantly down regulated in the distal and proximal small intestinal mucosa of LTS mice $(P<0.05$, Fig. 6a, Supplemental Fig. 5). Likewise, IL- $1 \alpha$ and IL-1 $\beta$ showed similar reductions only in small intestines of LTS mice $(P<0.05)$ (Fig. 6a and b, Supplemental Fig. 5). In contrast, Amphiregulin which was reported to increase following intestinal tissue injury [52], was significantly reduced in colonic mucosa of LTS mice (Fig. 6, Supplemental Fig. 5).

\section{Discussion}

Potential role of specific LGG-upregulated metabolites in inflammatory response

Competitive exclusion of pathogens is thought to be one of the most important benefits of probiotics, but the mechanisms of exclusion are unknown. While we cannot discount other mechanisms like competition for nutrients and for binding sites, the remarkable change in fecal metabolome elicited by LGG suggests that secretion of certain metabolites may create a hostile luminal ecology for the pathobionts (Section 4, Supplemental Table 3D). Interestingly, here we found that LGG increases levels of 


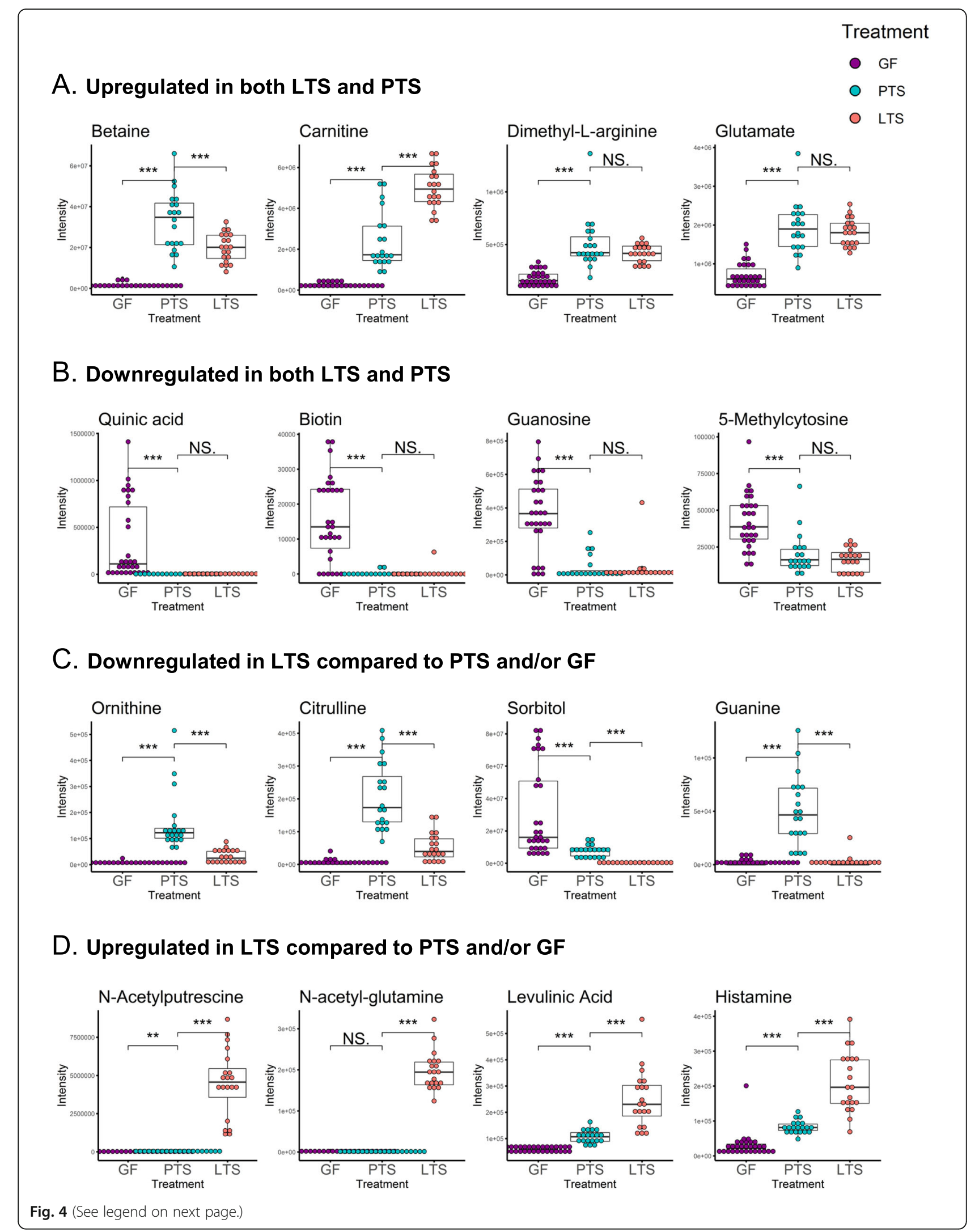


(See figure on previous page.)

Fig. 4 Patterns of regulation of identified metabolites in GF, PTS and LTS mice. a Box-whisker plots of representative upregulated metabolites in LTS and PTS mice compared to GF. b Box-whisker plots of representative downregulated metabolites in LTS and PTS mice compared to GF. c Box-whisker plots of representative downregulated metabolites in LTS compared to PTS and/or GF mice. $\mathbf{d}$ Box-whisker plots of representative upregulated metabolites in LTS compared to PTS and/or GF mice. ${ }^{*} P<0.05$; ${ }^{*} P<0.025$; ${ }^{* *} P<0.001$; NS $=$ not significant, $P>0.05$

levulinic acid, a potent anti-bacterial agent targeting mainly pathobionts as it inactivates Salmonella and verotoxigenic E. coli O157:H7 [53]. Levulinic acid is derived from bacteria-mediated degradation of cellulose as well as related carbohydrates and interestingly, can be made from fructose as well as $\mathrm{HCl}$. It is produced by L. bulgaricus in culture, is relatively nontoxic to host cells, and even supports growth of various Lactobacillus spp. Levulinic acid also is often used as a component in antiinflammatory medications, and a derivative, 5Aminolevulinic acid, reduced expression of proinflammatory cytokines TNF- $\alpha$, cyclooxygenase 2 , IL-1 $\beta$, and IL-6 as well as of iNOS and nitric oxide (NO) expression in the lipopolysaccharide-stimulated macrophage cell line RAW264.7 [54]. These findings on cytokine expression are similar to what we observed in the ileum of LGG-treated mice which showed remarkable reductions in TNF $\alpha$ and IL1 $\beta$ expression.

Other LGG-upregulated fecal metabolites also seem to have anti-inflammatory effects. The GI tract has many histamine receptors mediating the numerous functions of histamine, the most important of which are to regulate gastric acid secretion and intestinal inflammation [55]. Histamine is produced from L-histidine via histidine decarboxylase by some fermentative bacteria including Lactobacilli, and in fact, LGG has been shown to secrete histamine, explaining our findings of upregulation in LTS mice [56]. Intestinal luminal conversion of histidine to histamine by the probiotic $L$. reuteri activates the mouse histamine receptor $H 2 \mathrm{R}$ which subsequently results in suppression of IL- 6 and IL- $1 \beta$ expression as well as of acute inflammation in TNBSinduced colitis [56], and suppression of inflammatory TNF signaling via modulation of PKA and ERK pathways [57].

The AHR ligand indolelactic acid is specifically produced by several Lactobacillus spp., from tryptophan [58], and has been shown to interact with the AHR of a human immature small intestinal cell line to prevent transcription of the inflammatory cytokine IL-8 [59], to prevent production of IL- 6 in irradiated human keratinocytes [60], and even to scavenge free radicals [61]. Indolelactic acid produced by Lactobacillus spp. also seems to play an important role in regulating differentiation of $\mathrm{T}$ cells in the gut. $\mathrm{CD} 4^{+} \mathrm{CD} 8 \alpha \alpha^{+}$double-positive intraepithelial lymphocytes are a recently discovered class of intestinal $\mathrm{T}$ cells missing from GF mice but are believed to take part in immune tolerance. $L$. reuteri- synthesized indolelactic acid via the AHR receptor reprograms $\mathrm{CD}_{4}^{+}-\mathrm{T}$ cells to differentiate into immunoregulatory $\mathrm{CD} 4^{+} \mathrm{CD} 8 \alpha \alpha^{+}-\mathrm{T}$ cells [15]. Similarly, L. murinussynthesized indolelactic acid prevents salt-sensitive hypertension by reducing levels of pro-inflammatory IL17A-producing $\mathrm{CD}^{+} \mathrm{T}_{\mathrm{h}} 17$ cells [62].

The link between the other metabolites upregulated in LTS and inflammation is less clear. There are numerous acetylated amino acids upregulated in the LTS group. Acetylation of amino acids seems a unique contribution of and may be a biological signature of LGG. In many bacteria, $\mathrm{N}$-acetyltransferases move an acetyl group from acetyl-CoA to a large array of substrates, including naturally occurring polyamines like putrescine. Per KEGG pathway, this enzyme family is expressed in different strains of $L$. rhamnosus. The $\mathrm{N}$-acetylated form of $\mathrm{pu}-$ trescine is a metabolite of many bacteria, including Lactobacillus spp. A high level of $\mathrm{N}$-acetylputrescine was found in the feces of patients with ulcerative colitis and IBS [63]. N-acetyl-glutamine is a psychostimulant, nootropic, and anti-ulcer agent. L. plantarum increases production of $\mathrm{N}$-acetylated amino acids, including $\mathrm{N}$-acetylglutamine, to enhance Drosophila growth, thereby demonstrating an adaptive process by which a symbiotic bacterial strain increases its benefit to its animal host [64]. Ribitol, a pentose alcohol, is metabolized to teicholic acids used in the cell walls of Gram-positive bacteria.

\section{Potential effects of LGG-downregulated metabolites}

Many bacteria can synthesize arginine from glutamate via citrulline and ornithine [65] which is the precursor of polyamines like spermine also present in high concentrations in the feces of PTS mice. Arginine and ornithine are the main metabolic precursors of citrulline, and the conversion of arginine to citrulline releases NO. Ornithine, citrulline and arginine levels can indirectly but markedly affect intestinal function mainly through perturbations in levels of nitric oxide which regulates the permeability of the endothelium in the intestinal vasculature, and of epithelial cells in the mucosa [66]. In humans, high plasma levels of citrulline, as exhibited in our PTS mice, denote injury to the intestinal mucosal barrier and are correlated with high plasma levels of IL8 [67]. Unfortunately, there has been no study on the effect of luminal citrulline on the mucosal barrier.

Sorbitol can be made from glucose by aldose reductase [68]. It seems to be consumed modestly by PTS, but association with LGG effectively removed sorbitol from the feces, 







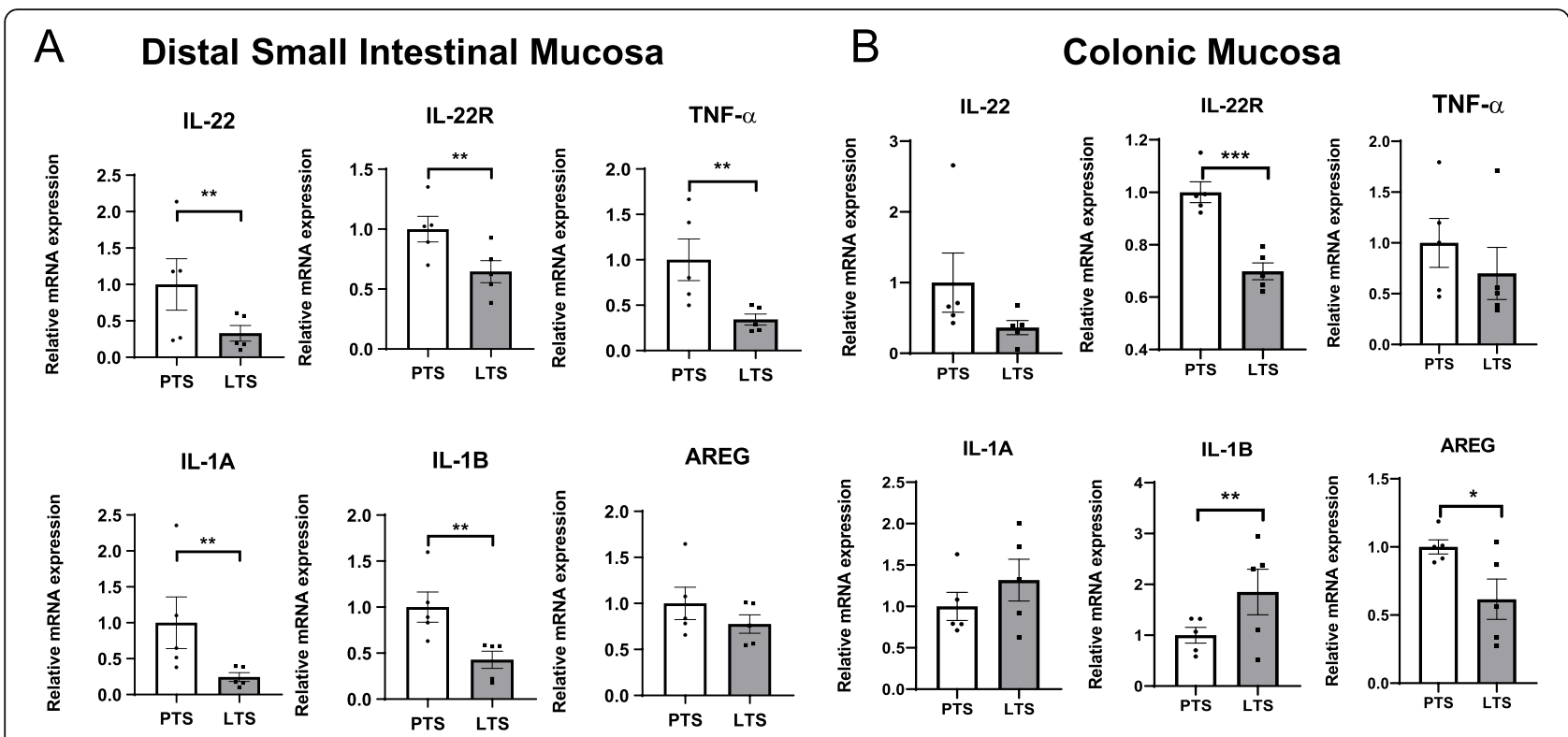

Fig. 6 LGG diminishes inflammatory response to pathobiont association. Relative mRNA expression of intestinal immune-related genes in distal intestinal (a) and colonic (b) mucosa in GF mice associated PTS or LTS (mean \pm SEM $(n=5)$. AREG = amphiregulin. ${ }^{*} P<0.05,{ }^{* *} P<0.01$

suggesting that LGG is a much more potent consumer of sorbitol (Fig. 4c). In fact, sorbitol has been shown to be utilized by many strains of L. rhamnosus [69], including LGG [70]. LGG expresses GutR, a factor that regulates the enzyme unit catabolizing sorbitol (http://regprecise.lbl.gov/ RegPrecise/genome.jsp?genome_id=506). Functionally, sorbitol is a sugar alcohol that works as a laxative by drawing water into the large intestine, thereby stimulating intestinal motility. Only a small amount of sorbitol is absorbed in the small intestine, and most enters the colon to elicit gastrointestinal effects. Patients with untreated celiac disease can present sorbitol malabsorption, which is an important cause for persisting disease symptoms. Overdose or improper use of sorbitol may cause severe gastrointestinal complications such as perforated colonic ulcers, ischemic colitis, colonic necrosis, and bleeding. These can likely be minimized by the intake of the probiotic LGG.

\section{Other significant VIP fecal metabolites}

Numerous Gram-positive and Gram-negative bacteria, including Lactobacillus spp. and other gut bacteria, synthesize both carnitine and betaine not only for metabolism but also for osmoprotection and bile tolerance which enables colonization of the gut lumen [71, 72]. Carnitine synthesis pathways are very highly conserved and are found in most fungi, bacteria, plants, and animals where carnitine shuttles long-chain fatty acids into the mitochondria so these can be oxidized to produce energy, and then transports toxic compounds out of the mitochondria to prevent their accumulation.
Many bacteria including Lactobacillus spp. can produce glutamate by fermentation [73]. Glutamate is important for two reasons. First, glutamate is a precursor to the important neurotransmitter $\gamma$-aminobutyric acid (GABA). Both gut bacteria and host cells can synthesize GABA through the decarboxylation of glutamate by glutamate decarboxylase whose encoding gene is present in gut microbiota. Luminal GABA from bacteria is then absorbed by the host small intestine for systemic use elsewhere in the body. Second, glutamate, along with glutamine, is a very important metabolic energy source of the intestinal mucosa. Luminal glutamate from dietary and bacterial sources is typically rapidly absorbed by the small intestine via the transporter EAAT3.

Nicotinate is a water-soluble B3 vitamin found in various animal and plant tissues. It is required by the body for the formation of coenzymes NAD+ and NADP+. It is not surprising that fecal levels of nicotinate are high in PTS and LTS mice, as the majority $(\sim 60 \%)$ of species in the gut microbiota, including members of all five major bacterial phyla, contain nicotinate biosynthesis pathways [74]. Nicotinate and members of the niacin family can be absorbed by the sodium-dependent monocarboxylate transporter SMCT1 in the host intestine. The high fecal glutamate and nicotinate levels in both PTS and LTS mice are likely due to the absence of EAAT3 and SMTC1 in the colon [75].

Although it accumulates in the feces, little is known about the synthesis of dimethyl-L-arginine. Its levels are physiologically important because it is an endogenous inhibitor of nitric oxide which regulates intestinal epithelial 
and endothelial permeability [66]. Interestingly, dimethylL-arginine concentrations increase in the serum of IBD patients [76]. Our novel findings here are likely very important as the intestinal capillaries of patients with IBD show microvascular endothelial dysfunction.

Because biotin, like many other " $\mathrm{B}$ " vitamins, is produced by a large number of gut microbiota species, it is surprising that this vitamin's concentration is reduced in the feces of PTS and LTS mice. The most likely explanation is that, unlike glutamate and nicotinate as mentioned above, biotin is not only more rapidly absorbed by the host, but its transporter, SMTV, is also expressed in the colon [77]. Bacteroides fragilis, L. helveticus and Campylobacter coli are common gut bacteria that can biosynthesize biotin [74, 78]. Thymidine, inosine and guanosine are nucleosides that, along with other nucleosides, nucleotides and nitrogenous bases, are assimilated rapidly by Lactobacillus spp. and other bacteria.

\section{LGG suppresses mucosal inflammatory response to pathobiont}

Proinflammatory cytokines like IL- $1 \alpha$, IL- $1 \beta$ and TNF- $\alpha$ seem to be consistently downregulated by LGG in both regions of the small intestine, but not in the colon. This is consistent with several reports that many Lactobacilli species reduce IL- $1 \beta$ and TNF- $\alpha$ levels in the presence of pathogenic bacteria [2, 7, 79]. Lactobacillus spp. are primarily located in the small intestine where they exert their influence on the gut microbiota and on the host mucosa [80]. We and others have shown that LGG secretes histamine which many Lactobacilli use to activate the histamine receptor $\mathrm{H} 2 \mathrm{R}$ and inhibit TNF signaling and IL-1 $\beta$ expression $[56,57]$, perhaps by interfering with NF-kB signaling [2]. Paralleling our results from LGG, other Lactobacilli also produce indoles and indole derivatives which reduce TNF- $\alpha$, IL-1 $\beta$ and other pro-inflammatory cytokines as well as alter IL-22 signaling [14, 81].

Our results extend previous findings showing several probiotic Lactobacillus species other than LGG modestly inhibiting $P$. acnes growth in vitro [82]. The dramatic inhibitory effect of LGG on $P$. acnes growth may be mediated by LGG metabolites, LGG-induced inhibition of bacterial adhesion, or LGG-induced acidification of the lumen [83], and can be translated in vivo as indicated by LGG exclusion of $P$. acnes and $T$. sanguinis in the gut lumen. It will be interesting to determine in future work the mechanisms underlying LGG competitive exclusion and/or growth inhibition of commensal bacteria with pathobiont potential.

\section{Conclusions}

The main findings of this study are that (1) P. acnes and $T$. sanguinis abundances are competitively inhibited by LGG, (2) the fecal metabolome of pathobionts is markedly altered by LGG, and (3) the inflammatory tone of the intestinal mucosa can be modified by LGG. An ancillary finding indicates that extraction of metabolites from feces is optimal when the volume of the extraction buffer is normalized to fecal sample abundance prior to analysis. While PTS is not particularly representative of naturally-found gut microbiomes, this study has been narrowly focused on metabolomic changes of these pathobionts/pathogens as a function of Lactobacillus administration. There is a need for additional studies to address the generalizability of these findings.

\section{Materials and methods \\ Mice}

For the first series of experiments involving optimization of metabolite extraction and normalization (Fig. 1a), eight-week old female and male C57BL/6 germ-free (GF) mice were purchased from Charles Rivers Laboratories (Wilmington, MA, USA). Following arrival, GF mice $(n=3)$ were unpacked and housed singly in autoclaved gnotobiotic cages on ventilated racks (Allentown, PA) at a temperature-controlled gnotobiotic facility at Rutgers New Jersey Medical School. GF mice were maintained on a 12:12 light-dark cycle and fed an autoclaved non-purified diet throughout the study (Supplemental Table 1A).

For the second set of experiments, eight-week old female C57BL/6 GF mice $(n=10)$ were purchased from Charles Rivers Laboratories. These mice were shipped in a sterilized GF container containing an autoclaved, nonpurified diet and water. All GF mice were then maintained on a 12:12 light-dark cycle and given ad libitum access to a double-irradiated, purified diet (Research Diet, New Brunswick, NJ) (Supplemental Table 1B) and autoclaved water throughout the study. On designated days, feces were collected from 08:00 AM - 10:00 AM. Animal procedures and protocols were conducted in accordance with the Rutgers University Institutional Animal Care and Use Committee.

\section{Administration of PBS and LGG}

Lactobacillus rhamnosus GG (LGG) (ATCC 53103) was purchased from American Tissue Culture Collection (ATCC, Manassas, Virginia, USA). LGG was cultured at $37^{\circ} \mathrm{C}$ in MRS broth (Fisher Scientific, Pittsburgh, PA, USA) following the manufacturer's protocol. The cultured broth was then centrifuged at $1200 \mathrm{~g}$ for $10 \mathrm{~min}$ to pellet the LGG. On Day 0 (Fig. 2a), a cohort of GF mice were gavaged with PBS, and another cohort with $200 \mu \mathrm{l}$ of LGG $\left(10^{8} \mathrm{CFU} / \mathrm{ml}\right)$ resuspended in the same PBS solution. The $P$. acnes, $T$. sanguinis, and $S$. aureus were identified from fecal samples of the PBS mice through bacterial $16 \mathrm{~S}$ sequencing. Since $P$. acnes was virtually 
eliminated by LGG in vivo, we then tested LGG inhibitory effects on P. acnes (ATCC 51277) in vitro.

\section{Fecal collection and DNA extraction}

Mice were placed in an empty autoclaved glass beaker and one fresh fecal pellet was transferred to a sterile 2 $\mathrm{ml}$ round-bottom tube (Nalgene, Rochester, NY) on dry ice and stored at $-80^{\circ} \mathrm{C}$ until further analysis. For DNA extraction, lysis buffer was adjusted by fecal weight and up to $800 \mu \mathrm{l}$ was added per bead-containing tube following the manufacturer's instructions using Purelink Microbiome DNA Purification Kit (Invitrogen, Carlsbad, CA, USA). Samples were homogenized (30 s) in a bead homogenizer (Thermo Fisher, Waltham, MA) and centrifuged at $14000 \mathrm{~g}(5 \mathrm{~min})$, and the supernatants were collected in a $1.5 \mathrm{~mL}$ microcentrifuge tube (Eppendorf, Hauppauge, NY). Samples were transferred to a spin column for purification and eluted in $100 \mu$ l elution buffer (Invitrogen by Thermo Fisher). DNA concentrations were determined by spectrophotometry (Nanodrop, Wilmington, DL). The purified DNA was used for bacterial $16 \mathrm{~S}$ rRNA qPCR to monitor potential contamination. For bacterial 16S rRNA gene sequencing, DNA of feces from GF mice was extracted using QIAamp DNA Stool Mini kit following the manufacturer's instructions (Qiagen Ltd., Strasse, Germany) for quality control.

\section{$16 \mathrm{~S}$ rRNA gene sequencing analysis}

Sequencing of the amplicon libraries was carried out using Ion Torrent ${ }^{\mathrm{Tm}}$ Personal Genome Machine $\mathrm{e}^{\mathrm{Tw}}$ (PGM) system (PrimBio Research Institute, Philadelphia, PA) and the Oxford Nanopore MinION platform [84]. In order to calculate downstream diversity measures (alpha and beta diversity indices), $16 \mathrm{~S}$ rRNA Operational Taxonomic Units (OTUs) were determined at $\geq 97 \%$ sequence homology.

\section{Quantitative real-time PCR (qPCR)}

LGG and $P$. acnes was quantified in fecal samples by quantitative real-time PCR using Agilent AriaMx Realtime PCR system. The following primers: LGG forward, 5'-CGCCCTTAACAGCAGTCTTC-3', reverse, 5'GCCCTCCGTATGCTTAAACC-3' and P.acnes forward, 5 ' -ATACGTAGGGTGCGAGCGTTGTCC-3', reverse, $\quad 5$ '-TGGTGTTCCTCCTGATATCTGCGC-3' were used. The $\mathrm{qPCR}$ reaction was performed using SYBR Green (Thermo Fisher) as follows: $95^{\circ} \mathrm{C}$ for $5 \mathrm{~min}$, followed by 45 cycles at $95^{\circ} \mathrm{C}$ for $10 \mathrm{~s}, 65^{\circ} \mathrm{C}$ for $15 \mathrm{~s}$, and $72^{\circ} \mathrm{C}$ for $15 \mathrm{~s}$, and a final extension at $95^{\circ} \mathrm{C}$ for $5 \mathrm{~min}$, $65^{\circ} \mathrm{C}$ for $1 \mathrm{~min}$, and $98^{\circ} \mathrm{C}$ for $30 \mathrm{~s}$. For $16 \mathrm{~S}$ rRNA quantitative PCR, primers that amplify gene encoding $16 \mathrm{~S}$ rRNA from all bacterial groups were employed in the amplification reaction: 16S rRNA forward 5'-AGAGTT TGAT CCTGGCTCAG-3' and reverse 5'-GACGGGCG
GT GWGTRCA-3'. No significant bacterial amplicon was detected in pre-inoculation fecal samples, i.e., under GF condition, when analyzed by qPCR using universal primers to amplify bacterial $16 \mathrm{~S}$ rRNA gene [85]. Here, the average Cq of fecal $16 \mathrm{~S}$ rRNA was $30.8 \pm 0.4$ before colonization, then $14.8 \pm 0.2$ for PTS and $14.1 \pm 0.2$ for LTS 5 days after colonization, consistent with studies showing absence and presence of bacteria [85].

\section{Extraction of metabolites and normalization of results}

Three normalization procedures were experimentally evaluated: (1) no normalization (using raw feature data), (2) "Post-LC-MS" and (3) "Pre-LC-MS" normalization, as depicted in Fig. 1 (a and d). Frozen feces were weighed on a calibrated scale and placed on ice. The polar metabolites were extracted with a mixture of acetonitrile/methanol/water (40:40:20) containing $0.1 \mathrm{M}$ formic acid (extraction buffer). For "Post-LC-MS" (Fig. 1a), equal volumes $(500 \mu \mathrm{l})$ of the extraction buffer were added to all fecal samples regardless of the fecal weight, and the resulting metabolite level was normalized by fecal weight post LC-MS data acquisition. For "Pre-LCMS" (Fig. 1d, normalization prior to LC-MS data acquisition), the volume of extraction buffer was adjusted by fecal weight prior to the extraction procedure. After addition of the extraction buffer to the feces, samples were then sonicated $\left(4{ }^{\circ} \mathrm{C}, 30 \mathrm{~s}\right)$ and centrifuged $(17,000$ g, $2 \mathrm{~min}$ ). After centrifugation, the supernatant collected $(150 \mu \mathrm{l})$ was diluted by four-fold with the extraction buffer prior to LC-MS analysis.

\section{LC-MS metabolomics analysis and metabolites identification}

The LC-MS analysis was performed on hydrophilic interaction chromatography coupled with electrospray ionization to the Q Exactive PLUS hybrid quadrupoleorbitrap mass spectrometer (Thermo Scientific) as previously described [86]. The LC separation was performed on a XBridge BEH Amide column $(150 \mathrm{~mm} \times 2.1 \mathrm{~mm}$, $2.5 \mu \mathrm{m}$ particle size, Waters, Milford, MA) using a gradient of solvent A $\left(95 \% / 5 \% \mathrm{H}_{2} \mathrm{O} /\right.$ acetonitrile with $20 \mathrm{mM}$ ammonium acetate and $20 \mathrm{mM}$ ammonium hydroxide, $\mathrm{pH} 9.4)$, and solvent $\mathrm{B}\left(20 \% / 80 \% \mathrm{H}_{2} \mathrm{O} /\right.$ acetonitrile with $20 \mathrm{mM}$ ammonium acetate and $20 \mathrm{mM}$ ammonium hydroxide, $\mathrm{pH}$ 9.4). The gradient was $0 \mathrm{~min}, 100 \% \mathrm{~B} ; 3 \mathrm{~min}$, $100 \%$ B; $3.2 \mathrm{~min}, 90 \%$ B; $6.2 \mathrm{~min}, 90 \%$ B; $6.5 \mathrm{~min}, 80 \%$ B; $10.5 \mathrm{~min}, 80 \% \mathrm{~B} ; 10.7 \mathrm{~min}, 70 \% \mathrm{~B} ; 13.5 \mathrm{~min}, 70 \% \mathrm{~B} ; 13.7$ min, $45 \% \mathrm{~B} ; 16 \mathrm{~min}, 45 \% \mathrm{~B} ; 16.5 \mathrm{~min}, 100 \% \mathrm{~B} ; 22 \mathrm{~min}$, $100 \% \mathrm{~B}$. The flow rate was $300 \mu \mathrm{L} / \mathrm{min}$. Injection volume was $5 \mu \mathrm{L}$ and column temperature $25^{\circ} \mathrm{C}$. Each sample was analyzed twice in both negative and positive ionization mode with a resolution of 70,000 at $\mathrm{m} / \mathrm{z} 200$. The automatic gain control target was $3 \times 10^{6}$. The maximum injection time was $50 \mathrm{~ms}$. Scan range was $75-$ 
1000. The MS2 spectra were collected from pooled samples under ddMS2 mode. The targeted metabolite data analysis was performed in MAVEN [87]. The compound identification was based on the accurate mass and the retention time learned from in-house chemical collection which includes 344 metabolites. The untargeted analysis was performed in Compound Discoverer (Thermo Scientific).

\section{Autoaggregation and coaggregation}

Propionibacterium acnes (ATCC 51277) was purchased from American Tissue Culture Collection (ATCC, Manassas, Virginia, USA). P. acnes was cultured in AnaeroGRO Chopped Meat Glucose Broth (Hardy Diagnostics, Catalog No. AG19H) at $37^{\circ} \mathrm{C}$ in an anerobic chamber. For autoaggregation experiments, $P$. acnes and LGG were harvested, washed with PBS, and re-suspended in $4 \mathrm{~mL}$ of blank meat broth respectively. The suspensions were then vortexed and incubated at room temperature for $48 \mathrm{~h}$. The autoaggregation percentage was determined using the following equation: Autoaggregation $(\%)=\left[\left(A_{0}-A_{t}\right) / A_{0}\right] \times 100$, where $A_{0}$ represents the absorbance $\left(\mathrm{OD}_{600}\right)$ at time $\mathrm{t}=0 \mathrm{~h}$ and $\mathrm{A}_{\mathrm{t}}$ represents the absorbance $\left(\mathrm{OD}_{600}\right)$ at time $\mathrm{t}=48 \mathrm{~h}$. For coaggregation experiments, equal volumes $(2 \mathrm{~mL})$ each of $P$. acnes and LGG were prepared, mixed by vortexing, incubated at room temperature for $48 \mathrm{~h}$ then absorbance $\left(\mathrm{OD}_{600}\right)$ values were measured. The coaggregation percentage was determined using the following equation: Coaggregation $(\%)=\left[\left\{\left(\mathrm{A}_{P \text {. acnes }}+\mathrm{A}_{\mathrm{LGG}}\right) / 2-\mathrm{A}_{P \text {. acnes }+\mathrm{LGG}}\right\} /\left\{\left(\mathrm{A}_{P}\right.\right.\right.$. acnes $\left.\left.\left.+\mathrm{A}_{\mathrm{LGG}}\right) / 2\right\}\right] \times 100$, Where $\mathrm{A}_{P \text {. acnes }}$ and $\mathrm{A}_{\mathrm{LGG}}$ represents the absorbance $\left(\mathrm{OD}_{600}\right)$ of $P$. acnes and LGG respectively at time $\mathrm{t}=48 \mathrm{~h}$ as mentioned in the autoaggregation test and $\mathrm{A}_{P}$. acnes + LGG represents the absorbance $\left(\mathrm{OD}_{600}\right)$ of $P$. acnes and LGG combined and incubated for $48 \mathrm{~h}$.

All tests for autoaggregation and coaggregation was repeated four times and the data were represented as mean \pm SEM. The zone of clearance was measured as the distance from the meniscus of the solution to the end of a clear solution.

\section{Collection and analysis of intestinal tissues for expression of immune-related genes}

At the end of the experiment, mice were anesthetized intraperitoneally $(0.2 \mathrm{ml} / 100 \mathrm{~g}$ body weight) with ketamine $(20 \mathrm{mg} / \mathrm{ml})$ and xylazine $(2.5 \mathrm{mg} / \mathrm{ml})$. The intestine was quickly removed and divided into three segments: proximal $(\sim 18 \mathrm{~cm})$ and distal $(\sim 18 \mathrm{~cm})$ small intestine and colon $(\sim 6 \mathrm{~cm})$. Segments were rinsed with ice-cold Dulbecco's phosphate-buffered saline (DPBS; Life Technologies, Carlsbad, CA, USA), and opened longitudinally in fresh ice-cold DPBS to expose the mucosa. These were then placed on the surface of an ice-cold petri dish and the entire mucosa was scraped off with precooled microscopic slides. Mucosal scrapes $(n=5)$ were stored in 1.5 $\mathrm{ml}$ clean Eppendorf tubes at $-70{ }^{\circ} \mathrm{C}$ until analysis.

The intestinal tissue mucosa was thawed on ice, homogenized using the Bullet Blender centrifuge (Next Advance, Atkinson, NH, USA) and total RNA was isolated from the intestinal mucosa using RNeasy Mini Kit (Qiagen, CA, USA). cDNA was synthesized using Bio-Rad iScript $^{\text {tw }}$ RT Supermix (Bio-Rad, Hercules, CA). The screening of immune-related gene expression was performed by qPCR using SYBR Green Master Mix (ThermoFisher, Waltham, MA, USA) and primers of target genes are listed in Supplemental Table 2. Gene expression was normalized to the housekeeping gene, $\beta$-actin.

\section{Statistical analysis}

Data are presented as mean \pm SEM, $(n=5$ except for data obtained for non-normalized and post LC-MS normalized). The bacterial OTUs (operational taxonomic units) using $16 \mathrm{~S}$ profiling of Ion Torrent PGM platform were used for the calculation of diversity dissimilarities [88]. Beta diversity of distance metrics were estimated UniFrac distances and followed by Bray-Curtis analysis to represent taxon-based difference within bacterial communities from different sample groups. The calculated data was defined by clustering using Principal Coordinate Analysis (PCoA) to assess patterns in the different sample groups. For metabolites analysis, Principal component analysis (PCA) and partial least squares discriminant analysis (PLS-DA) were performed to visualize differences in patterns of fecal metabolite levels between PTS and LTS or between the pre-gavage GF condition and each of the two groups [89]. We then calculated the VIP (variable's importance (influence) on the projection) scores of each metabolite based on the PLS-DA [90]. The VIP summarizes the contribution of a specific metabolite associated with either PTS or LTS colonization. A VIP score cut-off of > 1.2 was used as a variable selection based on discriminatory power, since VIP $>1$ is most influential for the model shown [48].

Student's t-test was performed to elicit statistically significant differences of the two sample groups in metabolites data analysis between GF and PTS, or PTS and LTS. For the qPCR data in Fig. 2, statistical significances were assessed by two-way ANOVA and Tukey's post-hoc test. The metabolomics datasets were analyzed by pattern recognition methods using MetaboAnalyst 4.0. Multivariate statistical analysis was conducted using MetaboAnalyst 4.0 PCA and PLS-DA were performed to find difference between metabolite profiles [47]. 


\section{Abbreviations}

AHR: Aryl hydrocarbon receptor; DSS: Dextran sodium sulfate; GF: Germ-free; IL-1: Interleukin 1; LGG: Lactobacillus rhamnosus GG; LC-MS: Liquid chromatography-mass spectrometry; LTS: Lactobacillus rhamnosus GG plus Propionibacterium acnes, Turicibacter sanguinis, and Staphylococcus aureus; OTU: Operational taxonomic unit; PCA: Principal component analysis; PCoA: Principal coordinate analysis; PLS-DA: Partial least squares discriminant analysis; PTS: Propionibacterium acnes, Turicibacter sanguinis, and Staphylococcus aureus; TNF-a: Tumor necrosis factor-alpha; VIP: Variable's importance in the projection

\section{Supplementary Information}

The online version contains supplementary material available at https://doi. org/10.1186/s12866-021-02178-2

Additional file 1: Supplementary Table 1A. Nutrient composition of autoclavable rodent pellets used in experiments optimizing the metabolite extraction procedure. Supplementary Table 1B. Nutrient composition of the diet used in the LTS and PTS experiment.

Supplementary Table 2. Primer sequences used in RT-qPCR of mouse genes. Supplementary Table $\mathbf{3 A}$. VIP scores and regulation of fecal metabolites in LTS compared to PTS mice. Supplementary Table 3B. VIP scores and regulation of fecal metabolites in GF compared to PTS mice. Supplementary Table $\mathbf{3 C}$. VIP scores and regulation of fecal metabolites in GF compared to LTS mice. Supplementary Table 3D. Highly significant VIP metabolites that are up or downregulated with PTS relative to GF, with LTS relative to GF, and LTS relative to PTS fall into four categories: (1) those that are upregulated in both PTS and LTS, (2) downregulated in both PTS and LTS, (3) upregulated in PTS or downregulated in LTS, and (4) upregulated in LTS. Supplementary Table 4. Accurate m/z and relative intensity values of peaks in the ms 2 spectra of unidentified metabolites.

Additional file 2: Supplementary Fig. 1. Experimental design and clinical parameters (A) Schematic diagram of experimental plan (B) Body weights as a function of age of mice before and after gavage with PTS or LTS (C) Diet consumption by mice over the course of the study.

Supplementary Fig. 2. Abundance of pathobionts when coassociated with LGG. Propionibacterium (A), Lactobacillus (B), Turicibacter (C), and Staphylococcus (D) abundance was determined in the feces from PTS $(n=5)$ and LTS $(n=5)$ mice by 165 amplicon profiling. Supplementary

Fig. 3. Unsupervised principal component analysis (PCA) of fecal metabolites from GF, PTS, and LTS mice. Supplementary Fig. 4 Accurate mass and MS2 spectra of unidentified metabolites.

Supplementary Fig. 5. Relative mRNA expression of intestinal immunerelated genes in mucosa of proximal small intestine in GF mice associated PTS or LTS.

\section{Acknowledgments}

We are grateful to Mr. Joshua Guardia for help in experiments and the staff of Comparative Medicine Resources, Rutgers University for help in establishing our germ-free colonies. Yuling He was supported by Guangxi Overseas Study Program by Guangxi Education Department.

\section{Authors' contributions}

J.K. conducted experiments, analyzed data, helped with experimental design, maintained animals, and wrote the initial draft of the manuscript; I.B. conducted experiments, analyzed data, helped with experimental design, revised the manuscript, helped address reviewer comments and compiled raw data for journal repository; S.B. conducted experiments, helped with experimental design, and revised the manuscript; R.S. analyzed data and revised the manuscript, D.H., I.N., A.B., Y.H., L.K. helped conduct experiments and analyzed data; N.G., X.S., R.P.F. conceptualized the project, designed the experiment, then finalized the submitted and revised manuscript. The author(s) read and approved the final manuscript.

\section{Funding}

Supported by NIH Grants R01-AT010243 (NG, RPF), R01DK102934 and R01DK119198 (NG), F31 DK121428 (SB), NSF Grant No. IOS 1754783 (RPF), ACS RSG-15-060-01-TBE (NG), NSF Grant 1952823 (NG). Services, results and/ or products in support of the research project were generated by the Rutgers Cancer Institute of New Jersey Metabolomics Shared Resource, supported, in part, with funding from NCI-CCSG P30CA072720-5923.

\section{Availability of data and materials}

The $16 \mathrm{~S}$ rRNA sequencing data generated in this study are deposited in SRA under accession number: PRJNA655706. The metabolomics data generated in this study are deposited in the MassIVE database under project MSV000085927.

\section{Declarations}

Ethics approval and consent to participate

Animal procedures, experimental protocols and methods were carried out in accordance with relevant guidelines and regulations, and were approved by the Rutgers University Institutional Animal Care and Use Committee, under Protocol No. 999900771. The study was carried in compliance with the ARRIVE guidelines (https://arriveguidelines.org) for the reporting of animal experiments.

\section{Consent for publication}

Not applicable.

\section{Competing interests}

All authors declare that they have no competing interests.

\section{Author details}

${ }^{1}$ Department of Pharmacology, Physiology and Neurosciences, Medical Science Building, New Jersey Medical School, Rutgers University, Newark, NJ 07103, USA. ²Department of Biological Sciences, Life Science Center, Rutgers University, Newark, NJ 07102, USA. ${ }^{3}$ Comparative Medicine Resources, Rutgers University, Newark, NJ 07103, USA. ${ }^{4}$ Department of Medicine, Clinical Academic Building, Robert Wood Johnson Medical School, Rutgers University, New Brunswick, NJ 08901, USA. ${ }^{5}$ Present address: Geriatric Endocrinology Division, The First Affiliated Hospital of Guangxi Medical University, Nanning, Guangxi, China. ${ }^{6}$ Department of Marine and Coastal Sciences, Rutgers University, 71 Dudley Rd, New Brunswick, NJ 08901, USA.

Received: 24 January 2021 Accepted: 25 February 2021

Published online: 03 June 2021

\section{References}

1. Jiang Y, Yang G, Meng F, Yang W, Hu J, Ye L, et al. Immunological mechanisms involved in probiotic-mediated protection against Citrobacter rodentium-induced colitis. Benefic Microbes. 2016;7(3):397-407. https://doi. org/10.3920/BM2015.0119.

2. Donato KA, Gareau MG, Wang YJ, Sherman PM. Lactobacillus rhamnosus GG attenuates interferon-\{gamma\} and tumour necrosis factor-alpha-induced barrier dysfunction and pro-inflammatory signalling. Microbiology. 2010; 156(Pt 11):3288-97. https://doi.org/10.1099/mic.0.040139-0.

3. Vong L, Lorentz RJ, Assa A, Glogauer M, Sherman PM. Probiotic Lactobacillus rhamnosus inhibits the formation of neutrophil extracellular traps. J Immunol. 2014;192(4):1870-7. https://doi.org/10.4049/jimmunol.13 02286.

4. Vigsnaes LK, van den Abbeele P, Sulek K, Frandsen HL, Steenholdt C, Brynskov J, et al. Microbiotas from UC patients display altered metabolism and reduced ability of LAB to colonize mucus. Sci Rep. 2013;3(1):1110. https://doi.org/10.1038/srep01110.

5. Segers ME, Lebeer S. Towards a better understanding of Lactobacillus rhamnosus GG--host interactions. Microb Cell Factories. 2014;13(Suppl 1):S7.

6. Levkovich T, Poutahidis T, Smillie C, Varian BJ, Ibrahim YM, Lakritz JR, et al. Probiotic bacteria induce a 'glow of health'. PLoS One. 2013;8(1):e53867. https://doi.org/10.1371/journal.pone.0053867.

7. Foster LM, Tompkins TA, Dahl WJ. A comprehensive post-market review of studies on a probiotic product containing Lactobacillus helveticus R0052 and Lactobacillus rhamnosus R0011. Benefic Microbes. 2011;2(4):319-34 https://doi.org/10.3920/BM2011.0032.

8. Takamura T, Harama D, Fukumoto S, Nakamura Y, Shimokawa N, Ishimaru K, et al. Lactobacillus bulgaricus OLL1181 activates the aryl hydrocarbon receptor pathway and inhibits colitis. Immunol Cell Biol. 2011:89(7):817-22 https://doi.org/10.1038/icb.2010.165. 
9. Claes IJ, Lebeer S, Shen C, Verhoeven TL, Dilissen E, De Hertogh G, et al. Impact of lipoteichoic acid modification on the performance of the probiotic Lactobacillus rhamnosus GG in experimental colitis. Clin Exp Immunol. 2010; 162(2):306-14. https://doi.org/10.1111/j.1365-2249.2010.04228.x.

10. Kankainen M, Paulin L, Tynkkynen S, von Ossowski I, Reunanen J, Partanen $P$, et al. Comparative genomic analysis of Lactobacillus rhamnosus GG reveals pili containing a human- mucus binding protein. Proc Natl Acad Sci U S A. 2009;106(40):17193-8. https://doi.org/10.1073/pnas.0908876106.

11. Velez MP, Petrova MI, Lebeer S, Verhoeven TL, Claes I, Lambrichts I, et al. Characterization of MabA, a modulator of Lactobacillus rhamnosus GG adhesion and biofilm formation. FEMS Immunol Med Microbiol. 2010;59(3): 386-98. https://doi.org/10.1111/j.1574-695X.2010.00680.x.

12. Bauerl C, Perez-Martinez G, Yan F, Polk DB, Monedero V. Functional analysis of the p40 and p75 proteins from Lactobacillus casei BL23. J Mol Microbiol Biotechnol. 2010;19(4):231-41. https://doi.org/10.1159/000322233.

13. Nermes M, Kantele JM, Atosuo TJ, Salminen S, Isolauri E. Interaction of orally administered Lactobacillus rhamnosus GG with skin and gut microbiota and humoral immunity in infants with atopic dermatitis. Clin Exp Allergy. 2011; 41(3):370-7. https://doi.org/10.1111/j.1365-2222.2010.03657.x.

14. Zelante T, lannitti RG, Cunha C, De Luca A, Giovannini G, Pieraccini G, et al. Tryptophan catabolites from microbiota engage aryl hydrocarbon receptor and balance mucosal reactivity via interleukin-22. Immunity. 2013;39(2):37285. https://doi.org/10.1016/j.immuni.2013.08.003.

15. Cervantes-Barragan L, Chai JN, Tianero MD, Di Luccia B, Ahern PP, Merriman J, et al. Lactobacillus reuteri induces gut intraepithelial CD4(+ )CD8alphaalpha(+) T cells. Science. 2017;357(6353):806-10. https://doi.org/1 $0.1126 /$ science.aah5825.

16. Zakostelska Z, Kverka M, Klimesova K, Rossmann P, Mrazek J, Kopecny J, et al. Lysate of probiotic Lactobacillus casei DN-114 001 ameliorates colitis by strengthening the gut barrier function and changing the gut microenvironment. PLoS One. 2011;6(11):e27961. https://doi.org/10.1371/ journal.pone.0027961.

17. Zubiria MG, Gambaro SE, Rey MA, Carasi P, Serradell MLA, Giovambattista A. Deleterious metabolic effects of high fructose intake: the preventive effect of Lactobacillus kefiri administration. Nutrients. 2017;9(5):E470.

18. Wang $C$, Zhu Y, Li F, Huang $L$. The effect of Lactobacillus isolates on growth performance, immune response, intestinal bacterial community composition of growing Rex rabbits. J Anim Physiol Anim Nutr (Berl). 2017;101(5):e1-e13. https://doi.org/10.1111/jpn.12629.

19. Su Y, Chen X, Liu M, Guo X. Effect of three lactobacilli with strain-specific activities on the growth performance, faecal microbiota and ileum mucosa proteomics of piglets. J Anim Sci Biotechnol. 2017;8(1):52. https://doi.org/1 0.1186/s40104-017-0183-3.

20. Almeida A, Mitchell AL, Boland M, Forster SC, Gloor GB, Tarkowska A, et al. A new genomic blueprint of the human gut microbiota. Nature. 2019; 568(7753):499-504. https://doi.org/10.1038/s41586-019-0965-1.

21. Willing BP, Van Kessel AG. Intestinal microbiota differentially affect brush border enzyme activity and gene expression in the neonatal gnotobiotic pig. J Anim Physiol Anim Nutr (Berl). 2009;93(5):586-95. https://doi.org/1 0.1111/j.1439-0396.2008.00841.x.

22. Willing BP, Van Kessel AG. Enterocyte proliferation and apoptosis in the caudal small intestine is influenced by the composition of colonizing commensal bacteria in the neonatal gnotobiotic pig. J Anim Sci. 2007; 85(12):3256-66. https://doi.org/10.2527/jas.2007-0320

23. Perez-Munoz ME, Bergstrom K, Peng V, Schmaltz R, Jimenez-Cardona R, Marsteller $\mathrm{N}$, et al. Discordance between changes in the gut microbiota and pathogenicity in a mouse model of spontaneous colitis. Gut Microbes. 2014; 5(3):286-95. https://doi.org/10.4161/gmic.28622.

24. Pasternak AJ, Hamonic GM, Van Kessel A, Wilson HL. Postnatal regulation of MAMDC4 in the porcine intestinal epithelium is influenced by bacterial colonization. Phys Rep. 2016;4(21).

25. Auchtung TA, Holder ME, Gesell JR, Ajami NJ, Duarte RT, Itoh K, et al. Complete Genome Sequence of Turicibacter sp. Strain H121, Isolated from the Feces of a Contaminated Germ-Free Mouse. Genome Announc. 2016: $4(2)$.

26. Achermann Y, Goldstein EJ, Coenye T, Shirtliff ME. Propionibacterium acnes: from commensal to opportunistic biofilm-associated implant pathogen. Clin Microbiol Rev. 2014;27(3):419-40. https://doi.org/10.1128/CMR.00092-13.

27. Mollerup S, Friis-Nielsen J, Vinner L, Hansen TA, Richter SR, Fridholm H, et al. Propionibacterium acnes: disease-causing agent or common contaminant?
Detection in diverse patient samples by next-generation sequencing. J Clin Microbiol. 2016;54(4):980-7. https://doi.org/10.1128/JCM.02723-15.

28. Perry A, Lambert P. Propionibacterium acnes: infection beyond the skin. Expert Rev Anti-Infect Ther. 2011;9(12):1149-56. https://doi.org/10.1586/ eri.11.137.

29. O'Neill CA, Monteleone G, McLaughlin JT, Paus R. The gut-skin axis in health and disease: a paradigm with therapeutic implications. Bioessays. 2016; 38(11):1167-76. https://doi.org/10.1002/bies.201600008.

30. Montalban-Arques A, Wurm P, Trajanoski S, Schauer S, Kienesberger S, Halwachs B, et al. Propionibacterium acnes overabundance and natural killer group 2 member D system activation in corpus-dominant lymphocytic gastritis. J Pathol. 2016;240(4):425-36. https://doi.org/10.1002/path.4782.

31. Gunathilake MN, Lee J, Choi IJ, Kim Yl, Ahn Y, Park C, et al. Association between the relative abundance of gastric microbiota and the risk of gastric cancer: a case-control study. Sci Rep. 2019;9(1):13589. https://doi.org/10.103 8/s41598-019-50054-x.

32. Cuiv PO, Klaassens ES, Durkin AS, Harkins DM, Foster L, McCorrison J, et al. Draft genome sequence of Turicibacter sanguinis PC909, isolated from human feces. J Bacteriol. 2011;193(5):1288-9. https://doi.org/10.1128/JB.0132 8-10.

33. Rossi G, Pengo G, Caldin M, Palumbo Piccionello A, Steiner JM, Cohen ND, et al. Comparison of microbiological, histological, and immunomodulatory parameters in response to treatment with either combination therapy with prednisone and metronidazole or probiotic VSL\#3 strains in dogs with idiopathic inflammatory bowel disease. PLoS One. 2014;9(4):e94699. https:// doi.org/10.1371/journal.pone.0094699.

34. Jones-Hall YL, Kozik A, Nakatsu C. Ablation of tumor necrosis factor is associated with decreased inflammation and alterations of the microbiota in a mouse model of inflammatory bowel disease. PLoS One. 2015;10(3): e0119441. https://doi.org/10.1371/journal.pone.0119441.

35. Zhong Y, Nyman M, Fak F. Modulation of gut microbiota in rats fed high-fat diets by processing whole-grain barley to barley malt. Mol Nutr Food Res. 2015;59(10):2066-76. https://doi.org/10.1002/mnfr.201500187.

36. Fung TC, Vuong HE, Luna CDG, Pronovost GN, Aleksandrova AA, Riley NG, et al. Intestinal serotonin and fluoxetine exposure modulate bacterial colonization in the gut. Nat Microbiol. 2019:4(12):2064-73. https://doi.org/1 0.1038/s41564-019-0540-4.

37. Kadariya J, Smith TC, Thapaliya D. Staphylococcus aureus and staphylococcal food-borne disease: an ongoing challenge in public health. Biomed Res Int. 2014;2014:827965.

38. Sannasiddappa TH, Costabile A, Gibson GR, Clarke SR. The influence of Staphylococcus aureus on gut microbial ecology in an in vitro continuous culture human colonic model system. PLoS One. 2011;6(8):e23227. https:// doi.org/10.1371/journal.pone.0023227.

39. Piewngam $P$, Zheng $Y$, Nguyen TH, Dickey SW, Joo HS, Villaruz AE, et al. Pathogen elimination by probiotic Bacillus via signalling interference. Nature. 2018;562(7728):532-7. https://doi.org/10.1038/s41586-018-0616-y.

40. Zeaki N, Johler S, Skandamis PN, Schelin J. The role of regulatory mechanisms and environmental parameters in staphylococcal food poisoning and resulting challenges to risk assessment. Front Microbiol. 2019;10:1307. https://doi.org/10.3389/fmicb.2019.01307.

41. Karpievitch $\mathrm{W}$, Polpitiya AD, Anderson GA, Smith RD, Dabney AR. Liquid chromatography mass spectrometry-based proteomics: biological and technological aspects. Ann Appl Stat. 2010;4(4):1797-823. https://doi.org/1 $0.1214 / 10-A O A S 341$.

42. Wu WK, Chen CC, Panyod S, Chen RA, Wu MS, Sheen LY, et al. Optimization of fecal sample processing for microbiome study - the journey from bathroom to bench. J Formos Med Assoc. 2019;118(2):545-55. https://doi. org/10.1016/j.jfma.2018.02.005.

43. Rabinowitz JD, Kimball E. Acidic acetonitrile for cellular metabolome extraction from Escherichia coli. Anal Chem. 2007;79(16):6167-73. https:// doi.org/10.1021/ac070470c

44. Hauser A, Eisenmann P, Muhle-Goll C, Luy B, Dotsch A, Graf D, et al. Efficient Extraction from Mice Feces for NMR Metabolomics Measurements with Special Emphasis on SCFAs. Metabolites. 2019;9(3).

45. Kong $\mathrm{H}$, Dai W. Xu G: [advances of metabolite identification in liquid chromatography-mass spectrometry based metabolomics]. Se Pu. 2014; 32(10):1052-7. https://doi.org/10.3724/sp.j.1123.2014.05017.

46. Xu W, Chen D, Wang N, Zhang T, Zhou R, Huan T, et al. Development of high-performance chemical isotope labeling LC-MS for profiling the human 
fecal metabolome. Anal Chem. 2015;87(2):829-36. https://doi.org/10.1021/a c503619q.

47. Chong J, Soufan O, Li C, Caraus I, Li S, Bourque G, et al. MetaboAnalyst 4.0: towards more transparent and integrative metabolomics analysis. Nucleic Acids Res. 2018;46(W1):W486-94. https://doi.org/10.1093/nar/gky310.

48. Magnusson MK, Isaksson S, Ohman L. The anti-inflammatory immune regulation induced by butyrate is impaired in inflamed intestinal mucosa from patients with ulcerative colitis. Inflammation. 2019.

49. Shi $\mathrm{D}$, Allewell NM, Tuchman M. The N-Acetylglutamate synthase family: structures, function and mechanisms. Int J Mol Sci. 2015;16(6):13004-22. https://doi.org/10.3390/ijms160613004.

50. Sharpatyi VA. Radiation chemistry of biopolymers. 1st ed. Boca Raton, FL: CRC Press; 2006

51. Naranjo Pinta M, Montoliu I, Aura AM, Seppanen-Laakso T, Barron D, Moco S. In vitro gut metabolism of [U-(13) C]-Quinic acid, the other hydrolysis product of Chlorogenic acid. Mol Nutr Food Res. 2018;62(22):e1800396. https://doi.org/10.1002/mnfr.201800396.

52. Monticelli LA, Osborne LC, Noti M, Tran SV, Zaiss DM, Artis D. IL-33 promotes an innate immune pathway of intestinal tissue protection dependent on amphiregulin-EGFR interactions. Proc Natl Acad Sci U S A. 2015;112(34):10762-7. https://doi.org/10.1073/pnas.1509070112.

53. Zhao T, Zhao P, Doyle MP. Inactivation of Salmonella and Escherichia coli O157:H7 on lettuce and poultry skin by combinations of levulinic acid and sodium dodecyl sulfate. J Food Prot. 2009;72(5):928-36. https://doi.org/1 0.4315/0362-028X-72.5.928.

54. Sugiyama Y, Hiraiwa Y, Hagiya Y, Nakajima M, Tanaka T, Ogura SI. 5Aminolevulinic acid regulates the immune response in LPS-stimulated RAW 264.7 macrophages. BMC Immunol. 2018;19(1):41.

55. Wechsler JB, Szabo A, Hsu CL, Krier-Burris RA, Schroeder HA, Wang MY, et al. Histamine drives severity of innate inflammation via histamine 4 receptor in murine experimental colitis. Mucosal Immunol. 2018;11(3):861-70. https:// doi.org/10.1038/mi.2017.121.

56. Gao C, Major A, Rendon D, Lugo M, Jackson V, Shi Z, et al. Histamine H2 Receptor-Mediated Suppression of Intestinal Inflammation by Probiotic Lactobacillus reuteri. mBio. 2015;6(6):e01358-15.

57. Thomas CM, Hong T, van Pijkeren JP, Hemarajata P, Trinh DV, Hu W, et al. Histamine derived from probiotic Lactobacillus reuteri suppresses TNF via modulation of PKA and ERK signaling. PLoS One. 2012;7(2):e31951. https:// doi.org/10.1371/journal.pone.0031951.

58. Roager HM, Licht TR. Microbial tryptophan catabolites in health and disease Nat Commun. 2018;9(1):3294. https://doi.org/10.1038/s41467-018-05470-4.

59. Meng D, Sommella E, Salviati E, Campiglia P, Ganguli K, Djebali K, et al. Indole-3-lactic acid, a metabolite of tryptophan, secreted by Bifidobacterium longum subspecies infantis is anti-inflammatory in the immature intestine. Pediatr Res. 2020;88(2):209-17. https://doi.org/10.1038/s41390-019-0740-x.

60. Aoki-Yoshida A, Ichida K, Aoki R, Kawasumi T, Suzuki C, Takayama Y. Prevention of UVB-induced production of the inflammatory mediator in human keratinocytes by lactic acid derivatives generated from aromatic amino acids. Biosci Biotechnol Biochem. 2013;77(8):1766-8. https://doi.org/1 $0.1271 / \mathrm{bbb} .120979$.

61. Suzuki Y, Kosaka M, Shindo K, Kawasumi T, Kimoto-Nira H, Suzuki C. Identification of antioxidants produced by Lactobacillus plantarum. Biosci Biotechnol Biochem. 2013;77(6):1299-302. https://doi.org/10.1271/bbb.121006.

62. Wilck N, Matus MG, Kearney SM, Olesen SW, Forslund K, Bartolomaeus H, et al. Salt-responsive gut commensal modulates TH17 axis and disease. Nature. 2017;551(7682):585-9. https://doi.org/10.1038/nature24628.

63. Le Gall G, Noor SO, Ridgway K, Scovell L, Jamieson C, Johnson IT, et al. Metabolomics of fecal extracts detects altered metabolic activity of gut microbiota in ulcerative colitis and irritable bowel syndrome. J Proteome Res. 2011;10(9):4208-18. https://doi.org/10.1021/pr2003598.

64. Martino ME, Joncour $P$, Leenay $R$, Gervais $H$, Shah M, Hughes $S$, et al. Bacterial Adaptation to the Host's Diet Is a Key Evolutionary Force Shaping Drosophila-Lactobacillus LESymbiosis. Cell Host Microbe. 2018;24(1):109-+.

65. Xu Y, Liang Z, Legrain C, Ruger HJ, Glansdorff N. Evolution of arginine biosynthesis in the bacterial domain: novel gene-enzyme relationships from psychrophilic Moritella strains (Vibrionaceae) and evolutionary significance of N-alpha-acetyl ornithinase. J Bacteriol. 2000;182(6):1609-15. https://doi. org/10.1128/JB.182.6.1609-1615.2000.

66. Kanwar S, Wallace JL, Befus D, Kubes P. Nitric oxide synthesis inhibition increases epithelial permeability via mast cells. Am J Phys. 1994;266(2 Pt 1): G222-9.
67. van Vliet MJ, Tissing WJ, Rings EH, Koetse HA, Stellaard F, Kamps WA, et al. Citrulline as a marker for chemotherapy induced mucosal barrier injury in pediatric patients. Pediatr Blood Cancer. 2009;53(7):1 188-94. https://doi. org/10.1002/pbc.22210.

68. Jans AW, Grunewald RW, Kinne RK. Pathways for the synthesis of sorbitol from 13C-labeled hexoses, pentose, and glycerol in renal papillary tissue. Magn Reson Med. 1989;9(3):419-22. https://doi.org/10.1002/mrm.191009031 5.

69. Ceapa C, Davids M, Ritari J, Lambert J, Wels M, Douillard FP, et al. The variable regions of Lactobacillus rhamnosus genomes reveal the dynamic evolution of metabolic and host-adaptation repertoires. Genome Biol Evol. 2016;8(6):1889-905. https://doi.org/10.1093/gbe/evw123.

70. Jiang Q, Kainulainen V, Stamatova I, Korpela R, Meurman JH. Lactobacillus rhamnosus GG in experimental Oral biofilms exposed to different carbohydrate sources. Caries Res. 2018;52(3):220-9. https://doi.org/10.1159/ 000479380.

71. Meadows JA, Wargo MJ. Carnitine in bacterial physiology and metabolism. Microbiology. 2015;161(6):1161-74. https://doi.org/10.1099/mic.0.000080.

72. Kets EPW, Galinski EA, Debont JAM. Carnitine - a novel compatible solute in Lactobacillus-Plantarum. Arch Microbiol. 1994;162(4):243-8. https://doi.org/1 $0.1007 / B F 00301845$

73. Mazzoli R, Pessione E. The Neuro-endocrinological role of microbial glutamate and GABA signaling. Front Microbiol. 2016;7:1934.

74. Magnusdottir S, Ravcheev D, de Crecy-Lagard V, Thiele I. Systematic genome assessment of B-vitamin biosynthesis suggests co-operation among gut microbes. Front Genet. 2015;6:148.

75. Hu QX, Ottestad-Hansen S, Holmseth S, Hassel B, Danbolt NC, Zhou Y. Expression of glutamate transporters in mouse liver, kidney, and intestine. J Histochem Cytochem. 2018;66(3):189-202. https://doi.org/10.1369/00221 55417749828.

76. Owczarek D, Cibor D, Mach T. Asymmetric dimethylarginine (ADMA), symmetric dimethylarginine (SDMA), arginine, and 8-iso-prostaglandin F2alpha (8-iso-PGF2alpha) level in patients with inflammatory bowel diseases. Inflamm Bowel Dis. 2010;16(1):52-7. https://doi.org/10.1002/ibd.20994.

77. Said HM. Intestinal absorption of water-soluble vitamins in health and disease. Biochem J. 2011;437(3):357-72. https://doi.org/10.1042/BJ20110326.

78. Patel A, Shah N, Prajapati JB. Clinical application of probiotics in the treatment of helicobacter pylori infection--a brief review. J Microbiol Immunol Infect. 2014;47(5):429-37. https://doi.org/10.1016/j.jmii.2013.03.010.

79. Wu Y, Zhu C, Chen Z, Chen Z, Zhang W, Ma X, et al. Protective effects of Lactobacillus plantarum on epithelial barrier disruption caused by enterotoxigenic Escherichia coli in intestinal porcine epithelial cells. Vet Immunol Immunopathol. 2016;172:55-63. https://doi.org/10.1016/j.vetimm.2 016.03.005.

80. Walter J. Ecological role of lactobacilli in the gastrointestinal tract: implications for fundamental and biomedical research. Appl Environ Microbiol. 2008;74(16):4985-96. https://doi.org/10.1128/AEM.00753-08.

81. Shi J, Du P, Xie Q, Wang N, Li H, Smith EE, et al. Protective effects of tryptophan-catabolizing Lactobacillus plantarum KLDS 1.0386 against dextran sodium sulfate-induced colitis in mice. Food Funct. 2020;11(12): 10736-47. https://doi.org/10.1039/DOFO02622K.

82. Al-Ghazzewi FH, Tester RF. Effect of konjac glucomannan hydrolysates and probiotics on the growth of the skin bacterium Propionibacterium acnes in vitro. Int J Cosmet Sci. 2010;32(2):139-42. https://doi.org/10.1111/j.14 68-2494.2009.00555.x.

83. Hosseini A, Nikfar S, Abdollahi M. Probiotics use to treat irritable bowel syndrome. Expert Opin Biol Ther. 2012;12(10):1323-34. https://doi.org/10.151 7/14712598.2012.707179

84. Kerkhof LJ, Dillon KP, Haggblom MM, McGuinness LR. Profiling bacterial communities by MinlON sequencing of ribosomal operons. Microbiome. 2017;5(1):116. https://doi.org/10.1186/s40168-017-0336-9.

85. Fontaine CA, Skorupski AM, Vowles CJ, Anderson NE, Poe SA, Eaton KA. How free of germs is germ-free? Detection of bacterial contamination in a germ free mouse unit. Gut Microbes. 2015;6(4):225-33. https://doi.org/10.1 080/19490976.2015.1054596.

86. Su X, Chiles E, Maimouni S, Wondisford FE, Zong WX, Song C. In-source CID ramping and covariant ion analysis of hydrophilic interaction chromatography metabolomics. Anal Chem. 2020;92(7):4829-37. https://doi. org/10.1021/acs.analchem.9b04181. 
87. Melamud E, Vastag L, Rabinowitz JD. Metabolomic analysis and visualization engine for LC-MS data. Anal Chem. 2010;82(23):9818-26. https://doi.org/10.1 021/ac1021166.

88. Pylro VS, Roesch LF, Morais DK, Clark IM, Hirsch PR, Totola MR. Data analysis for 165 microbial profiling from different benchtop sequencing platforms. J Microbiol Methods. 2014;107:30-7. https://doi.org/10.1016/j.mimet.2014.08. 018

89. Worley B, Powers R. Multivariate analysis in metabolomics. Curr Metabolomics. 2013;1 (1):92-107. https://doi.org/10.2174/2213235X1130101 0092.

90. Gromski PS, Muhamadali H, Ellis DI, Xu Y, Correa E, Turner ML, et al. A tutorial review: metabolomics and partial least squares-discriminant analysis--a marriage of convenience or a shotgun wedding. Anal Chim Acta. 2015;879:10-23. https://doi.org/10.1016/j.aca.2015.02.012.

\section{Publisher's Note}

Springer Nature remains neutral with regard to jurisdictional claims in published maps and institutional affiliations.

Ready to submit your research? Choose BMC and benefit from:

- fast, convenient online submission

- thorough peer review by experienced researchers in your field

- rapid publication on acceptance

- support for research data, including large and complex data types

- gold Open Access which fosters wider collaboration and increased citations

- maximum visibility for your research: over $100 \mathrm{M}$ website views per year

At BMC, research is always in progress.

Learn more biomedcentral.com/submissions 